\title{
Genome-wide association screening and verification of potential genes associated with root architectural traits in maize (Zea mays $L$.) at multiple seedling stages
}

Abdourazak Alio Moussa ${ }^{1 *}$, Ajmal Mandozai ${ }^{1}$, Yukun Jin ${ }^{1}$, Jing Qu', Qi Zhang ${ }^{1}$, He Zhao ${ }^{1}$, Gulaqa Anwari ${ }^{1}$, Mohamed Abdelsamiaa Sayed Khalifa', Abraham Lamboro', Muhammad Noman², Yacoubou Bakasso', Mo Zhang ${ }^{1}$, Shuyan Guan ${ }^{1}$ and Piwu Wang ${ }^{1 *}$

\begin{abstract}
Background: Breeding for new maize varieties with propitious root systems has tremendous potential in improving water and nutrients use efficiency and plant adaptation under suboptimal conditions. To date, most of the previously detected root-related trait genes in maize were new without functional verification. In this study, seven seedling root architectural traits were examined at three developmental stages in a recombinant inbred line population (RIL) of 179 RILs and a genome-wide association study (GWAS) panel of 80 elite inbred maize lines through quantitative trait loci (QTL) mapping and genome-wide association study.

Results: Using inclusive composite interval mapping, 8 QTLs accounting for $6.44-8.83 \%$ of the phenotypic variation in root traits, were detected on chromosomes 1 (qRDW $v_{v 3}-1-1$ and $\left.q R D W / S D W_{v 3}-1-1\right), 2$ (qRBN $\left.v_{v 1}-2-1\right), 4\left(q S U A_{v 1}-4-1\right.$, $\mathrm{qSUA}_{\mathrm{v} 2}-4-1$, and $\mathrm{qROV} \mathrm{v}_{2}-4-1$ ), and 10 (qTRL $\left.\mathrm{v}_{\mathrm{v}}-10-1, \mathrm{qRBN}_{\mathrm{v} 1}-10-1\right)$. GWAS analysis involved three models (EMMAX, FarmCPU, and MLM) for a set of 1,490,007 high-quality single nucleotide polymorphisms (SNPs) obtained via whole genome next-generation sequencing (NGS). Overall, 53 significant SNPs with a phenotypic contribution rate ranging from 5.10 to $30.2 \%$ and spread all over the ten maize chromosomes exhibited associations with the seven root traits. 17 SNPs were repeatedly detected from at least two growth stages, with several SNPs associated with multiple traits stably identified at all evaluated stages. Within the average linkage disequilibrium (LD) distance of $5.2 \mathrm{~kb}$ for the significant SNPs, 46 candidate genes harboring substantial SNPs were identified. Five potential genes viz. Zm00001d038676, Zm00001d015379, Zm00001d018496, Zm00001d050783, and Zm00001d017751 were verified for expression levels using maize accessions with extreme root branching differences from the GWAS panel and the RIL population. The results showed significantly $(P<0.001)$ different expression levels between the outer materials in both panels and at all considered growth stages.
\end{abstract}

* Correspondence: abdoulrazakalio@gmail.com; peiwuw@163.com

${ }^{1}$ College of Agronomy, Plant Biotechnology Center, Jilin Agricultural University, 130118 Changchun, Jilin, China

Full list of author information is available at the end of the article

(c) The Author(s). 2021 Open Access This article is licensed under a Creative Commons Attribution 4.0 International License, which permits use, sharing, adaptation, distribution and reproduction in any medium or format, as long as you give appropriate credit to the original author(s) and the source, provide a link to the Creative Commons licence, and indicate if changes were made. The images or other third party material in this article are included in the article's Creative Commons licence, unless indicated otherwise in a credit line to the material. If material is not included in the article's Creative Commons licence and your intended use is not permitted by statutory regulation or exceeds the permitted use, you will need to obtain permission directly from the copyright holder. To view a copy of this licence, visit http://creativecommons.org/licenses/by/4.0/ The Creative Commons Public Domain Dedication waiver (http://creativecommons.org/publicdomain/zero/1.0/) applies to the data made available in this article, unless otherwise stated in a credit line to the data. 
Conclusions: This study provides a key reference for uncovering the complex genetic mechanism of root development and genetic enhancement of maize root system architecture, thus supporting the breeding of highyielding maize varieties with propitious root systems.

Keywords: Maize, root-related traits, GWAS, SNPs, Candidate genes, qRT-PCR

\section{Background}

Maize (Zea mays L.) is one of the most widely produced grain crops in the world [1]. With the fast-growing world population, improving the yield of corn has become an important target for breeders. The root system plays a primordial role in plant species growth and development and even productivity [2-4]. Plants rely on the root system for anchorage and the acquisition and absorption of nutrients essential for sustaining productivity [2]. As the place of plant and soil interactions, roots play a fundamental role in plant responses to biotic and abiotic stresses [5], and influence significantly many agronomically important traits, including drought and flood tolerance [6-8], root-lodging resistance [9], and nutrient use efficiency particularly nitrogen $(\mathrm{N})$, phosphorus(P), and calcium (Ca) under suboptimal growth conditions [10-13] and resource-challenging environments $[2,14,15]$. Important synchronizations were previously revealed between root growth especially shootborne roots with $\mathrm{N}$ uptake efficiency in maize $[15,16]$. Besides, some pieces of evidence support that high yielding maize varieties are supposed to have propitious root systems, which may efficiently sustain water and nutrients, resulting in increased yield [17] especially under limited water or nutrient availability [18]. Furthermore, grain yield was reported to be closely correlated with root related traits in the early stages of maize development [19]. Nevertheless, maize roots have received much less attention than shoot structures since they are hidden, complex, dynamic and greatly influenced by the soil environment $[15,20-22]$. Due to the challenge in achieving reliable root-related trait data from the field, characterizing crops such as maize with improved root system characteristics in the field remains still a major challenge to current plant biology $[18,23]$ and root trait phenotyping studies commonly use soil-less nutritive solutions [5]. However, it was previously indicated that plant growing systems that nearly mimic the soil media are more stable in mineral elements and environmental factors, and easier to operate for root morphological traits phenotyping in maize [24]. Therefore, to offer a better and robust tool for plant behavior prediction under field conditions, various experimental growing systems with soil-based substrates have been implemented [25-28]. Owing to the rapid progress in digitally automatic image analysis, root phenotypic data acquisition is becoming nowadays cheaper, quicker and more effective [29-34]. Thus, numerous software frameworks such as ARIA [31], EZ-Rhizo [35], Smart Root [36], WinRhizo [37], Optimas analysis software, Image J [38], Root Nav [32], IJ_Rhizo [39], Root System Analyzer [40], and Root Trace [41] have been broadly used for automated root traits measurements in a high throughput manner.

To date, several Quantitative Trait Loci (QTL) studies have been conducted to locate root-trait QTLs under various conditions of growth, at diverse developmental stages and involving various genetic populations [21, 22, 24]. Yet, due to low-density markers and large confidence intervals, the localizations of the identified QTLs were inconsistent among the different findings. Thus, further root studies were necessary to detect more chromosomal regions and ultimately identify consistent loci to further screen and verify candidate genes crucial for marker-assisted selection. By enabling the identification of essential loci at high-resolution, association studies have several advantages over conventional genetic mapping approaches for understanding the genetic basis of complex traits [42] like root traits in maize [2]. In the 21st century, genome-wide association studies (GWAS) have been auspiciously used as a high-throughput technique to analyzing the genetic basis for a variety of major crops [42], such as rice, sorghum, soybean, wheat, and maize essential for modern genetic studies [43]. Recently, Sanchez et al. [44] used 300 doubled haploid exotic introgression lines and found 39 SNPs for root architecture traits along with, multiple SNPs within candidate genes that displayed expression in maize seedling roots. In a GWAS analysis implying 14 days old maize seedlings generated from 384 inbred lines genotyped by sequencing (GBS), 268 SNPs associated with root morphological traits, along with 9 SNPs within one candidate gene region were reported [2]. Zaidi et al. [8] used a CIMMYT Asia panel involving 396 tropical maize lines. They revealed 67 SNPs associated with root structural traits under drought stress with many SNPs found within various candidate gene regions. However, to our knowledge no previous study has investigated the genetic basis of maize root architectural traits at multiple developmental stages with successful functional verification of associated candidate genes. Therefore, the objectives of this study were to (i) screen the existing phenotypic variability of root architectural traits within a maize elite germplasm at multiple seedling stages, (2) 
detect novel significant genomic regions throughout the whole genome and across stages associated with root architectural traits, and (3) identify and verify the expression of possible potential candidate genes.

\section{Results}

\section{Phenotypic analysis of root architectural traits}

From the mapping population, the evaluated root traits displayed large variations both in parental lines and their offspring at all the three stages (Table 1 and Additional file 1: Table S1). Analysis of variance related to the root trait performances of the two parental lines revealed significant to highly significant differences $(P<0.05 ; P<$ $0.01 ; P<0.001)$ for all the measured seedling traits and at all the three stages except RDW/SDW at V1 stage (Table 1). Comparing the two parents, P014 displayed significantly higher root trait performances than E1312 across the three stages (Table 1). This result shows the instantaneous nature of the development of the two parental root systems over time which confirms the pertinence of the three selected experimental time-points for root traits assessment. RBN and TRL exhibited the largest variations of 264.94 and $121.00 \mathrm{~cm}$, respectively (Table 1). Similar heritability and correlation patterns were also observed across stages. Heritability values ranged between 50.22 ( for RDW/SDW) and 99.96\% (for TRL). SUA and TRL exhibited the strongest positive significant correlations $(r=0.924 ; P<0.01)$ while RDW/ SDW showed very weak correlations with all other traits $(r=0.149 \sim 0.464 ; P<0.05 ; P<0.01$; Table 2$)$.

Similarly, substantial variation at all growth stages was observed within the GWAS population for all root traits evaluated (Table 3 and Additional file 2: Table S2). At stage V3, RBN and ROV showed the highest coefficients of variation of 77.16 and $76.47 \%$, respectively. Most root-related traits investigated nearly followed a normal distribution, somewhat skewed from left to right (Fig. 1). Moderate to high broad-sense heritability estimates were observed for all seedling traits and at all stages (Table 3). The highest value was observed from RBN (99.84\%) while the lowest one from ARD (43.20\%) (Table 3). Pearson correlation analysis was also performed to

Table 1 Descriptive statistics of the seven root-related traits within the mapping population at V1, V2 and V3 stages

\begin{tabular}{|c|c|c|c|c|c|c|c|c|c|c|c|}
\hline \multirow[t]{2}{*}{ Traits } & \multirow[t]{2}{*}{ Stage } & \multirow{2}{*}{$\begin{array}{l}\text { P014 } \\
\text { Mean }\end{array}$} & \multirow{2}{*}{$\begin{array}{l}\text { E1312 } \\
\text { Mean }\end{array}$} & \multirow[t]{2}{*}{ Sig. $^{a}$} & \multicolumn{7}{|l|}{ RILs } \\
\hline & & & & & Mean & $\pm \mathrm{SD}$ & Range & Skewness & Kurtosis & CV (\%) & $\overline{H^{2}(\%)}$ \\
\hline \multirow[t]{3}{*}{ RDW(g) } & V1 & 0.04 & 0.01 & * & 0.03 & 0.03 & 0.17 & 1.85 & 3.60 & 90.05 & 70.91 \\
\hline & V2 & 0.06 & 0.03 & $* *$ & 0.05 & 0.03 & 0.20 & 1.37 & 2.58 & 59.01 & 69.34 \\
\hline & V3 & 0.07 & 0.04 & $* *$ & 0.10 & 0.05 & 0.32 & 1.06 & 1.32 & 52.54 & 85.73 \\
\hline \multirow[t]{3}{*}{ RDW/SDW } & V1 & 0.87 & 0.38 & ns & 0.78 & 1.10 & 9.91 & 4.28 & 22.43 & 141.33 & 65.94 \\
\hline & V2 & 0.48 & 0.38 & $* *$ & 0.33 & 0.19 & 2.28 & 3.66 & 26.67 & 58.90 & 50.22 \\
\hline & V3 & 0.25 & 0.19 & * & 0.41 & 0.17 & 1.49 & 1.25 & 3.56 & 41.91 & 83.27 \\
\hline \multirow[t]{3}{*}{ TRL(cm) } & V1 & 73.79 & 36.86 & $* * *$ & 55.31 & 25.19 & 147.89 & 0.68 & 0.68 & 45.55 & 98.81 \\
\hline & V2 & 155.29 & 100.07 & $* * *$ & 123.57 & 57.78 & 418.32 & 1.46 & 4.41 & 46.76 & 99.24 \\
\hline & V3 & 216.63 & 107.19 & $* * *$ & 249.96 & 121.00 & 521.61 & 0.44 & -0.69 & 48.41 & 99.96 \\
\hline \multirow[t]{3}{*}{ SUA $\left(\mathrm{cm}^{2}\right)$} & V1 & 28.19 & 16.42 & $* * *$ & 19.32 & 9.75 & 66.03 & 1.17 & 2.93 & 50.48 & 99.31 \\
\hline & V2 & 40.69 & 35.71 & $* * *$ & 40.17 & 22.12 & 134.38 & 1.44 & 2.88 & 55.07 & 94.73 \\
\hline & V3 & 76.30 & 43.73 & $* *$ & 91.16 & 51.44 & 235.91 & 0.68 & -0.28 & 56.43 & 97.89 \\
\hline \multirow[t]{3}{*}{ ARD (mm) } & V1 & 1.13 & 1.00 & $* *$ & 0.94 & 0.20 & 1.46 & 1.22 & 4.07 & 21.46 & 97.74 \\
\hline & V2 & 2.31 & 1.16 & $* * *$ & 1.12 & 0.25 & 2.32 & 2.43 & 11.89 & 22.52 & 88.49 \\
\hline & V3 & 3.49 & 2.45 & $* * *$ & 1.38 & 0.45 & 4.57 & 4.22 & 28.76 & 32.69 & 95.46 \\
\hline \multirow[t]{3}{*}{$\mathrm{ROV}\left(\mathrm{cm}^{3}\right)$} & V1 & 1.15 & 0.63 & $* * *$ & 0.56 & 0.36 & 2.60 & 2.31 & 8.84 & 65.52 & 98.15 \\
\hline & V2 & 2.88 & 0.84 & $* * *$ & 1.13 & 0.73 & 4.38 & 1.29 & 1.91 & 64.32 & 96.66 \\
\hline & V3 & 3.08 & 1.79 & $* * *$ & 2.82 & 2.26 & 22.94 & 3.63 & 25.17 & 80.25 & 97.35 \\
\hline \multirow[t]{3}{*}{ RBN } & V1 & 51.33 & 28.33 & $* * *$ & 45.66 & 26.57 & 126.00 & 0.67 & 0.03 & 58.19 & 99.28 \\
\hline & V2 & 94.33 & 52.67 & $* * *$ & 105.88 & 93.84 & 1051.00 & 6.33 & 59.19 & 88.64 & 99.79 \\
\hline & V3 & 331.00 & 145.33 & $* * *$ & 264.94 & 362.10 & 4518.00 & 9.39 & 107.55 & 136.67 & 99.89 \\
\hline
\end{tabular}

$R D W$ root dry weight, $R D W / S D W$ root per shoot dry weight, $T R L$ total root length, SUA surface area, $A R D$ average root diameter, $R O V$ root volume, $R B N$ root branching number, SD Std. dev, $C V$ coefficient of variation, $H^{2}$ Broad-sense heritability

${ }^{a}$ level of significance via student-test with, ns no significant difference

*significantly different at $P<0.05$

**significantly different at $P<0.01$

*** significantly different at $P<0.001$ 
Table 2 Pearson correlation coefficients between the seven root-related traits within the mapping population at V1, V2 and V3 stages

\begin{tabular}{|c|c|c|c|c|c|c|}
\hline Traits & RDW & RDW/SDW & TRL & SUA & ARD & ROV \\
\hline \multicolumn{7}{|l|}{ V1 } \\
\hline RDW/SDW & $0.719^{* *}$ & & & & & \\
\hline TRL & $0.330^{* *}$ & 0.022 & & & & \\
\hline SUA & $0.389^{* *}$ & 0.081 & $0.886^{* *}$ & & & \\
\hline ARD & $0.194^{* *}$ & 0.050 & 0.055 & $0.261^{* *}$ & & \\
\hline ROV & $0.425^{* *}$ & 0.103 & $0.669^{* *}$ & $0.854^{* *}$ & $0.514^{* *}$ & \\
\hline RBN & $0.299^{* *}$ & 0.050 & $0.835^{* *}$ & $0.781^{* *}$ & 0.087 & $0.597^{* *}$ \\
\hline \multicolumn{7}{|l|}{ V2 } \\
\hline RDW/SDW & $0.648^{* *}$ & & & & & \\
\hline TRL & $0.659^{* *}$ & $0.270^{* *}$ & & & & \\
\hline SUA & $0.535^{* *}$ & $0.184^{* *}$ & $0.826^{* *}$ & & & \\
\hline ARD & 0.004 & 0.029 & 0.011 & $0.309^{* *}$ & & \\
\hline ROV & $0.459^{* *}$ & $0.156^{*}$ & $0.614^{* *}$ & $0.884^{* *}$ & $0.502^{* *}$ & \\
\hline RBN & $0.473^{* *}$ & $0.304^{* *}$ & $0.783^{* *}$ & $0.758^{* *}$ & 0.114 & $0.577^{* *}$ \\
\hline \multicolumn{7}{|l|}{ V3 } \\
\hline RDW/SDW & $0.675^{* *}$ & & & & & \\
\hline TRL & $0.771^{* *}$ & $0.412^{* *}$ & & & & \\
\hline SUA & $0.818^{* *}$ & $0.464^{* *}$ & $0.924^{* *}$ & & & \\
\hline ARD & 0.000 & 0.054 & -0.036 & 0.051 & & \\
\hline ROV & $0.639^{* *}$ & $0.353^{* *}$ & $0.680^{* *}$ & $0.843^{* *}$ & $0.247^{* *}$ & \\
\hline RBN & $0.310^{* *}$ & $0.149^{*}$ & $0.442^{* *}$ & $0.523^{* *}$ & $0.169^{*}$ & $0.800^{* *}$ \\
\hline
\end{tabular}

$R D W$ root dry weight, $R D W / S D W$ root per shoot dry weight, $T R L$ total root length, SUA surface area, ARD average root diameter, ROV root volume, $R B N$ root branching number

the symbol * and ${ }^{* *}$ indicate respectively, significance at $P<0.05$ and at $\mathrm{P}<0.01$

examine the phenotypic relationships among root related traits at each specified stage. Similar significant correlation patterns, but with a greater extent at later stages were detected (Table 4). In regards to all evaluated traits at all stages, ROV and SUA exhibited the strongest positive significant correlations $(r>90 \%, P<$ 0.01 ) while RDW/SDW is weakly correlated to all other traits $(r=-0.199 \sim 0.477 ; P<0.05 ; P<0.01$; Table 4$)$.

\section{QTL mapping}

The linkage map contained 4235 high-quality SNP markers covering a total length of $1514.57 \mathrm{cM}$ distributed for 10 linkage groups [45]. Using inclusive composite interval mapping method with $\mathrm{LOD} \geq 2.5$ as a threshold, a total of eight substantial QTLs with a phenotypic variance explained ranging from 6.44 to $8.83 \%$ were detected across the three stages (Table 5). The mapped QTLs were allocated to chromosomes 1, 2, 4 , and 10. Chromosome 4 contained the highest number of QTLs, with 3 QTLs detected while chromosomes 1, 2, and 10 contained between 1 and 2 QTLs (Table 5). Four
QTLs were detected at V1 while two QTLs where identified at both V2 and V3 stages. When examining the number of QTL inheriting parental favorable alleles, the alleles involved in increasing root characteristics at four loci belonged to the parent P014. Meanwhile, the paternal line E1312 contributed to the other four loci, underlying, therefore, the imperative implication of the two parents in root features discrimination. QTL clusters were identified on chromosome 1 and 10 at V3 and V1, respectively. The cluster on chromosome $1\left(\mathrm{qRDW}_{\mathrm{v} 3}-1\right.$ 1 and $\left.\mathrm{qRDW} / \mathrm{SDW}_{\mathrm{v} 3}-1-1\right)$ located within the marker interval Snp3292_Snp3298 was associated with RDW and RDW/SDW at the genetic region 92.5- $95.5 \mathrm{cM}$. The Cluster on chromosome $10\left(\mathrm{qRBN}_{\mathrm{v} 1}-10-1\right.$ and qTRL $\left._{\mathrm{v} 1}-10-1\right)$ detected within the marker interval Snp62466_Snp62578 was significantly associated to RBN and TRL and spanned 50.5-51.5 cM genetic region. This region harbored two candidate genes GRMZM2G116542 and GRMZM2G016477 predicted to encode a putative Spc97 / Spc98 family of spindle pole body (SBP) component and a putative leucine-rich repeat receptor-like protein kinase, respectively. The three QTLs detected on chromosome 4 (qSUA $_{\mathrm{v1}}-4-1$, $\mathrm{qSUA}_{\mathrm{v} 2}-4-1$, and $\left.\mathrm{qROV}_{\mathrm{v} 2}-4-1\right)$ were significantly associated with SUA and ROV and spanned the genetic region 89.5-102.5 cM. QTL qROV ${ }_{\mathrm{v} 2}-4-1 \quad(\mathrm{LOD}=3.43$, $\mathrm{PVE}=8.83 \%)$ associated with ROV was the most significant QTL detected in this study (Table 5). Interestingly, the gene model GRMZM2G068506 predicted to encode a Glucose-1-phosphate adenylyltransferase was found within this chromosomal region. The physical positions of all the detected QTLs are presented in Additional file 3: Table S3.

\section{NGS analysis results}

High-quality genomic data consisted of $3230.75 \mathrm{~Gb}$ with an average of $40.38 \mathrm{~Gb}$ per sample were obtained from the whole-genome sequencing of the 80 inbred maize lines. All related sequences were made available under the accession number PRJNA495031 in the Sequence Read Archive (https:/www.ncbi.nlm.gov/sra). The averages sequencing depth and coverage were 17.62 and $88.39 \%$, respectively. With reference to the B73 genome (RefGen_v3), the average similarity rate was $98.82 \%$.

\section{Population structure and linkage disequilibrium}

Based on phylogenetic and PCA analysis, the 80 inbred maize lines were subdivided into three subgroups (Fig. 2A, B). Subgroup 1 mainly included the Reid germplasm represented by PH09B and PH6WC maize inbred lines. Subgroup 2 included mainly the Chinese Lvda Red $\mathrm{Cob}$ and Tang Si Ping Tou germplasm as well as some tropical maize lines. Subgroup 3 comprised European and Lancaster germplasm, including Non-Reid maize 
Table 3 Descriptive statistics of seedling root related traits for the GWAS population at three stages

\begin{tabular}{|c|c|c|c|c|c|c|c|c|}
\hline Traits & Stage & Mean & $\pm \mathrm{SD}$ & Range & Skewness & Kurtosis & CV (\%) & $H^{2}(\%)$ \\
\hline \multirow[t]{3}{*}{ RDW (g) } & V1 & 0.06 & 0.02 & 0.15 & 0.39 & 2.24 & 40.82 & 94.07 \\
\hline & V2 & 0.10 & 0.06 & 0.31 & 0.97 & 0.49 & 62.46 & 84.68 \\
\hline & V3 & 0.12 & 0.07 & 0.34 & 0.82 & 0.33 & 55.73 & 71.89 \\
\hline \multirow[t]{3}{*}{ RDW/SDW } & V1 & 0.60 & 1.38 & 7.88 & 2.56 & 7.39 & 86.32 & 90.56 \\
\hline & V2 & 0.63 & 0.41 & 3.27 & 2.75 & 11.75 & 65.32 & 80.87 \\
\hline & V3 & 0.45 & 0.18 & 1.09 & 0.80 & 1.13 & 39.84 & 61.71 \\
\hline \multirow[t]{3}{*}{ TRL(cm) } & V1 & 94.29 & 48.98 & 261.42 & 0.93 & 0.64 & 51.95 & 97.87 \\
\hline & V2 & 195.30 & 134.37 & 699.97 & 1.41 & 2.45 & 68.80 & 99.52 \\
\hline & V3 & 305.10 & 184.01 & 780.26 & 0.71 & -0.33 & 60.31 & 99.51 \\
\hline \multirow[t]{3}{*}{ SUA $\left(\mathrm{cm}^{2}\right)$} & V1 & 32.90 & 16.67 & 79.18 & 0.95 & 0.54 & 50.68 & 96.17 \\
\hline & V2 & 70.28 & 52.53 & 251.41 & 1.34 & 1.89 & 74.74 & 98.67 \\
\hline & V3 & 107.91 & 66.56 & 294.09 & 0.66 & -0.42 & 61.68 & 99.30 \\
\hline \multirow[t]{3}{*}{ ARD (mm) } & V1 & 1.08 & 0.26 & 1.46 & 0.61 & 0.20 & 22.11 & 58.08 \\
\hline & V2 & 1.12 & 0.30 & 2.35 & 1.88 & 7.56 & 27.11 & 51.31 \\
\hline & V3 & 1.28 & 0.22 & 1.42 & 0.64 & 1.29 & 20.26 & 43.29 \\
\hline \multirow[t]{3}{*}{$\mathrm{ROV}\left(\mathrm{cm}^{3}\right)$} & V1 & 0.96 & 0.52 & 2.57 & 0.93 & 0.63 & 54.24 & 75.11 \\
\hline & V2 & 2.04 & 1.75 & 8.83 & 1.44 & 1.99 & 86.06 & 94.27 \\
\hline & V3 & 3.02 & 2.31 & 13.48 & 1.35 & 2.79 & 76.47 & 89.26 \\
\hline \multirow[t]{3}{*}{ RBN } & V1 & 69.47 & 45.45 & 201.00 & 1.23 & 1.15 & 65.43 & 98.51 \\
\hline & V2 & 169.39 & 132.69 & 642.00 & 1.47 & 2.30 & 78.34 & 99.28 \\
\hline & V3 & 275.04 & 212.22 & 864.00 & 1.18 & 0.75 & 77.16 & 99.84 \\
\hline
\end{tabular}

$R D W$ root dry weight, $R D W / S D W$ root per shoot dry weight, $T R L$ total root length, SUA surface area, $A R D$ average root diameter, $R O V$ root volume, $R B N$ root branching number, SD Std. dev, CV coefficient of variation, $H^{2}$ Broad-sense heritability

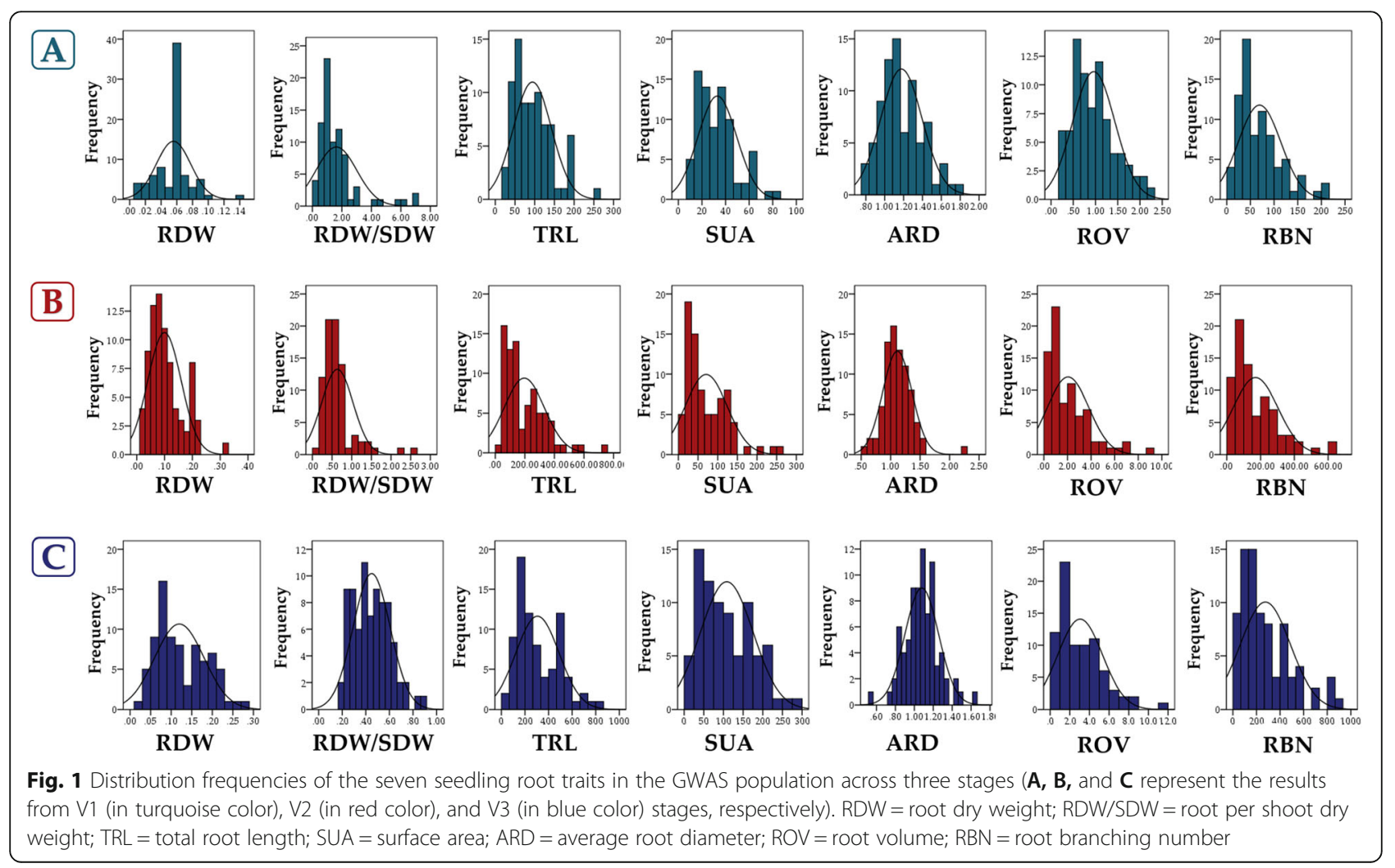


Table 4 Pearson correlations at three stages among all rootrelated traits for GWAS population

\begin{tabular}{|c|c|c|c|c|c|c|}
\hline Traits & RDW & RDW/SDW & TRL & SUA & ARD & ROV \\
\hline \multicolumn{7}{|l|}{ V1 } \\
\hline RDW/SDW & $0.509^{* *}$ & & & & & \\
\hline TRL & 0.115 & $-0.251^{*}$ & & & & \\
\hline SUA & 0.183 & $-0.224^{*}$ & $0.902^{* *}$ & & & \\
\hline ARD & 0.037 & 0.025 & $-0.303^{* *}$ & -0.031 & & \\
\hline ROV & 0.172 & -0.203 & $0.731^{* *}$ & $0.907^{* *}$ & $0.0262^{*}$ & \\
\hline RBN & 0.104 & -0.212 & $0.752^{* *}$ & $0.790^{* *}$ & -0.114 & $0.749^{* *}$ \\
\hline \multicolumn{7}{|l|}{ V2 } \\
\hline RDW/SDW & 0.101 & & & & & \\
\hline TRL & $0.788^{* *}$ & -0.183 & & & & \\
\hline SA & $0.822^{* *}$ & $-0.214^{*}$ & $0.941^{* *}$ & & & \\
\hline ARD & $0.381^{* *}$ & -0.127 & $0.247^{*}$ & $0.418^{* *}$ & & \\
\hline ROV & $0.816^{* *}$ & $-0.199^{*}$ & $0.857^{* *}$ & $0.970^{* *}$ & $0.542^{* *}$ & \\
\hline RBN & $0.634^{* *}$ & $-0.209^{*}$ & $0.837^{* *}$ & $0.852^{* *}$ & $0.378^{* *}$ & $0.814^{* *}$ \\
\hline \multicolumn{7}{|l|}{ v3 } \\
\hline RDW/SDW & $0.615^{* *}$ & & & & & \\
\hline TRL & $0.773^{* *}$ & $0.423^{* *}$ & & & & \\
\hline SUA & $0.843^{* *}$ & $0.477^{* *}$ & $0.870^{* *}$ & & & \\
\hline ARD & $0.394^{* *}$ & $0.215^{*}$ & 0.104 & $0.343^{* *}$ & & \\
\hline ROV & $0.813^{* *}$ & $0.438^{* *}$ & $0.704^{* *}$ & $0.933^{* *}$ & $0.532^{* *}$ & \\
\hline RBN & $0.719^{* *}$ & $0.451^{* *}$ & $0.900^{* *}$ & $0.806^{* *}$ & 0.100 & $0.649 * *$ \\
\hline
\end{tabular}

$R D W$ root dry weight, $R D W / S D W$ root per shoot dry weight, TRL total root length, SUA surface area, $A R D$ average diameter, ROV root volume, $R B N$ root branching number

the symbol ${ }^{*}$ and ${ }^{* *}$ indicate respectively, significance at $P<0.05$ and at $P<0.01$

inbred lines such as PHB1 $\mathrm{M}$ and Mo17. As shown in Fig. 3, the average decay distance of the LD across all chromosomes was about $5.2 \mathrm{~kb}$ at $\mathrm{r}^{2}=0.1$. Chromosome 1 with a distance of $10.7 \mathrm{~kb}$ showed the highest LD decay while the shortest decay distance $(3.7 \mathrm{~kb})$ was observed on chromosome 2 .

\section{GWAS for root architectural traits}

In this current analysis, three GWAS approaches including EMMAX, FarmCPU, and MLM were used to scan significant SNPs associated with seven root traits namely RDW, RDW/SDW, TRL, SUA, ARD, ROV, and RBN across three vegetative stages (V1, V2, and V3). The detailed list of all significant SNPs detected in this study and their associated genes is presented in Additional file 4: Table S4. SNPs identified within candidate genes or across at least two different stages/methods simultaneously were considered as reliable in this study. Hence, according to these criteria, 53 unique SNPs, along with 46 SNPs within candidate genes that exhibited significant associations with root morphological traits at the critical threshold of $-\log _{10}(P) \geq 6.0$ were obtained (Table 6; Fig. 4). These abovementioned SNPs were distributed all over the 10 maize chromosomes and individually explained between 5.10 and $30.2 \%$ of phenotypic variation (Table 6). When analyzing significant SNPs that were detected throughout different stages, 17 SNPs were repeatedly detected from at least two stages along with 3 stable SNPs scanned across all the three growth stages (Table 6; Fig. 4). Our study regarded these SNPs as of great interest for further breeding purposes. Comparing the results across the different GWAS approaches, 34, 19, and 1 SNPs were identified by EMMAX, FarmCPU, and MLM, respectively (Table 6; Fig. 4). The SNP with the lowest p-value was located on chromosome 7, position 58,218,452 $\left(-\log _{10}(P)=14.95, \mathrm{R}^{2}=30.2 \%\right)$, and was associated with RBN and SUA. This SNP was detected by FarmCPU stably across V1 and V3 stages. The SNP on chromosome $2\left(\mathrm{~S} 2 \_1707072,-\log _{10}(P)=8.36, \mathrm{R}^{2}=25.1 \%\right)$ was simultaneously detected by two different methods (EMMAX, MLM) at V2 stage. In regards of significant SNPs controlling multiple traits, two SNPs located on chromosomes 1 and 5 (S1_227871089, S5_82882718) were substantially linked to three root traits including ROV $\left(-\log _{10}(P)=6.06,14.10, \mathrm{R}^{2}=14 \%, 22.8 \%\right), \mathrm{RDW}\left(-\log _{10}(P\right.$

Table 5 Summary of root traits QTLs detected in P014 × E1312 population

\begin{tabular}{|c|c|c|c|c|c|c|c|c|}
\hline $\mathrm{QTL}^{\mathrm{a}}$ & Chr & Bin & Peak(cM) & Marker interval & Genetic interval(cM) & LOD & PVE $^{\mathrm{b}}$ (\%) & Add. ${ }^{\circ}$ \\
\hline $\mathrm{qRDW}_{\mathrm{v} 3^{-1-1}}$ & 1 & 1.05 & 95 & Snp3292_Snp3298 & $92.5-95.5$ & 2.51 & 6.74 & -0.01 \\
\hline qRDW/SDW v3 $^{-1-1}$ & 1 & 1.05 & 95 & Snp3292_Snp3298 & $92.5-95.5$ & 2.51 & 6.74 & -0.01 \\
\hline $\mathrm{qRBN}_{\mathrm{v} 1}-2-1$ & 2 & 2.10 & 15 & Snp16808_Snp16675 & $14.5-15.5$ & 2.51 & 6.44 & 6.38 \\
\hline$q S \cup A_{v 1}-4-1$ & 4 & 4.05 & 91 & Snp25452_Snp25434 & $89.5-91.5$ & 2.67 & 6.72 & 2.53 \\
\hline$q S \cup A_{v 2}-4-1$ & 4 & 4.05 & 102 & Snp26234_Snp26219 & $100.5-102.5$ & 3.03 & 7.75 & 5.97 \\
\hline $\mathrm{qROV}_{\mathrm{v} 2}-4-1$ & 4 & 4.05 & 96 & Snp25161_Snp25085 & $95.5-96.5$ & 3.43 & 8.83 & 0.21 \\
\hline $\mathrm{qTRL}_{\mathrm{v} 1}-10-1$ & 10 & $10.05-06$ & 51 & Snp62466_Snp62578 & $50.5-51.5$ & 2.66 & 6.77 & -6.65 \\
\hline $\mathrm{qRBN}_{\mathrm{V} 1}-10-1$ & 10 & $10.05-06$ & 51 & Snp62466_Snp62578 & $50.5-54.5$ & 2.73 & 7.16 & -6.81 \\
\hline
\end{tabular}

${ }^{a}$ The identified QTLs: the name contains trait initials, seedling growing stage, and the number of correspondent chromosome

${ }^{b}$ The percentages of phenotypic variation explained

'The QTL additive effect: positive values indicate that P014 provides increased alleles and negative ones indicate that E1312 alleles increased the trait 

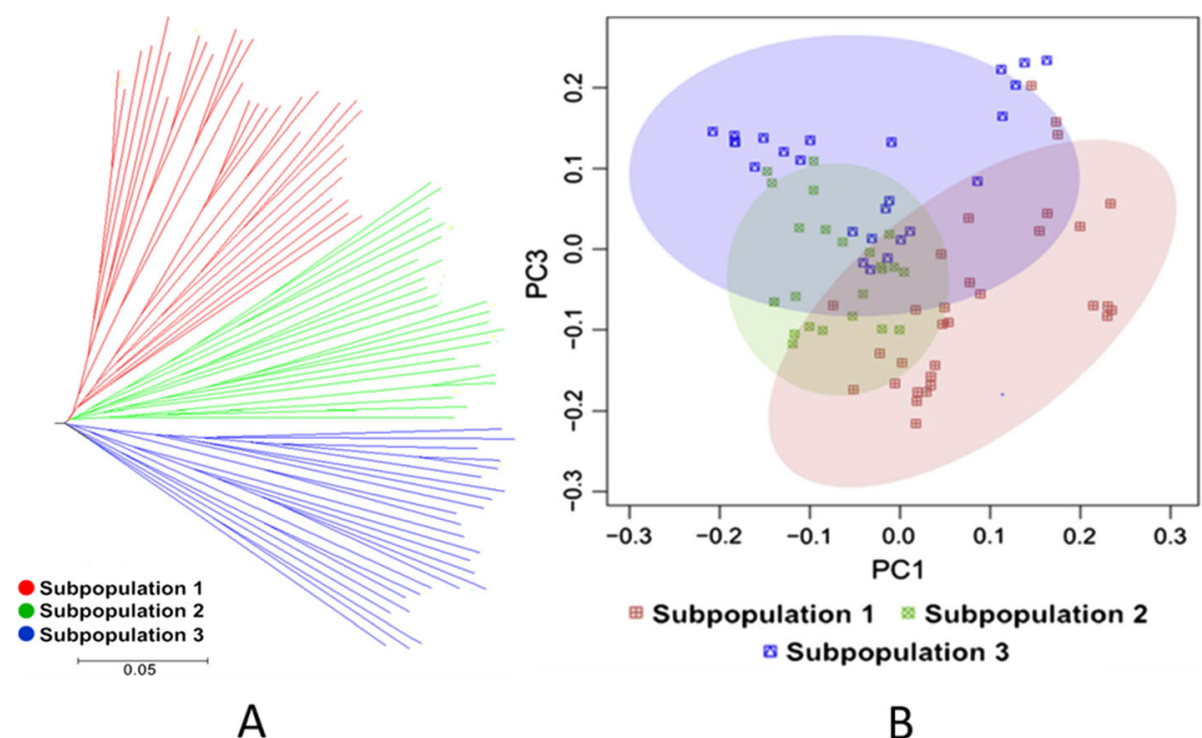

B

Fig. 2 Population structure of the 80 maize accessions: A Phylogenetic generated using TreeBeST, B Principal component analysis based on genome-wide complex trait analysis software tool (GCTA)

)$\left.=6.10,6.87, \mathrm{R}^{2}=14 \%, 6.3 \%\right)$, and SUA $\left(-\log _{10}(P)=\right.$ 7.02, $\left.14.10 \mathrm{R}^{2}=12.1 \%, 22.8 \%\right)$, respectively. Another SNP on chromosome 2 (S2_43293834, $-\log _{10}(P)=6.89$, $\mathrm{R}^{2}=11 \%$ ) was also significantly linked with three different root traits, including RBN, ROV and SUA. The Q-Q (quantile-quantile) plots of all traits at all stages are shown in Additional file 5: Figure S1.

\section{Candidate genes and functional annotations}

A total of 46 genes, along with 41 genes with SNPs inside, were found showing associations with the seven root architectural traits (Table 7 ). The candidate gene Zm00001d019766 was found only $5.54 \mathrm{~kb}$ away from the most significant SNP detected in this study located on chromosome 7 (S7_58218452) and associated with

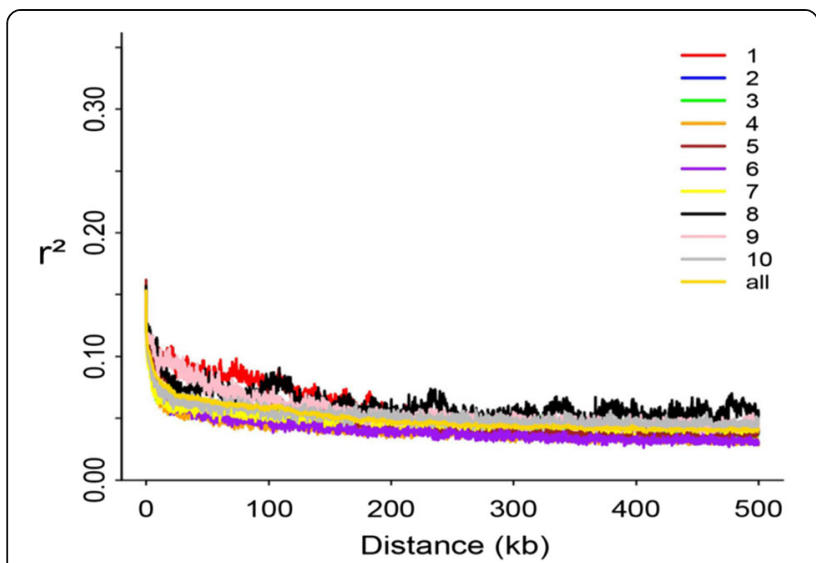

Fig. 3 Linkage disequilibrium decay across all 10 maize chromosomes within the 80 maize panel
RBN and SUA. This gene was predicted to encode a RING/U-box superfamily protein. The gene model Zm00001d032473 on chromosome 1 (S1_227871089 for ROV, RDW, and SUA located within exon of the candidate gene) was predicted to confer a nonsynonymous mutation associated with CDPK-related kinase 3. The candidate genes Zm00001d029482 (S1_ 72599741 and S1_72599769 within the candidate gene) and Zm00001d037546 (S6_128905260 and S6_ 128905254 within the candidate gene) located respectively on chromosomes 1 and 6 contained two significant markers found for two traits, TRL and RBN. The gene model $\mathrm{Zm00001d029482} \mathrm{was} \mathrm{predicted} \mathrm{to} \mathrm{encode}$ a NAD (P)-binding Rossmann-fold superfamily protein. Gene model Zm00001d005925 (SNP S2 192996724 for RBN located within the candidate gene) encodes a phosphoglucose isomerase protein with various pathways including GDP-mannose biosynthesis, gluconeogenesis I, glycolysis I (from glucose 6phosphate), starch biosynthesis, and sucrose biosynthesis I. Gene model Zm00001d017279 on chromosome 5 (SNP S5_191539297 for RDW located within the candidate gene) encodes a phenylalanine ammonia-lyase protein associated with transcinnamoyl-CoA biosynthesis pathways. Gene Zm00001d038676 located on chromosome 6 (SNP S6 162388475 for RBN located within the candidate gene) encodes xyloglucan 6-xylosyltransferase and xyloglucan glycosyltransferase associated with xyloglucan and biosynthesis. The details of all candidate genes associated with potential SNPs and the functional annotations are presented in Table 7. 
Table 6 Potential significant SNPs associated with root related traits

\begin{tabular}{|c|c|c|c|c|c|c|c|c|}
\hline Traits & Position & Method $^{a}$ & Stage & Chr & P-value & $-\log _{10}(P)$ & $R^{2}$ & Genotype \\
\hline ARD & $209,661,144$ & 2 & V2 & Chr3 & 8.51E-08 & 7.07 & 0.100 & $\mathrm{G} / \mathrm{A}$ \\
\hline ARD & $159,805,368$ & 2 & V2 & Chr6 & 8.32E-07 & 6.08 & 0.111 & $\mathrm{~T} / \mathrm{G}$ \\
\hline RBN & $72,599,741$ & 1 & $\mathrm{~V} 1, \mathrm{~V} 3$ & Chr1 & 3.31E-07 & 6.48 & 0.101 & $\mathrm{~T} / \mathrm{G}$ \\
\hline RBN & $127,352,056$ & 1 & V2 & Chr1 & $2.95 \mathrm{E}-07$ & 6.53 & 0.115 & $\mathrm{~T} / \mathrm{C}$ \\
\hline RBN & $1,707,072$ & 1,3 & V2 & Chr2 & 4.37E-09 & 8.36 & 0.251 & $\mathrm{G} / \mathrm{A}$ \\
\hline RBN & $192,996,724$ & 1 & V2 & Chr2 & 7.59E-08 & 7.12 & 0.151 & $C / A$ \\
\hline RBN & $195,707,091$ & 1 & V2 & Chr2 & $6.17 \mathrm{E}-08$ & 7.21 & 0.152 & $\mathrm{C} / \mathrm{T}$ \\
\hline RBN & $2,564,296$ & 1 & V2 & Chr2 & $1.86 \mathrm{E}-07$ & 6.73 & 0.108 & $\mathrm{C} / \mathrm{T}$ \\
\hline RBN & $32,824,965$ & 1 & V2 & Chr2 & $6.76 \mathrm{E}-08$ & 7.17 & 0.107 & $\mathrm{G} / \mathrm{C}$ \\
\hline RBN & $171,057,172$ & 2 & V2 & Chr3 & 4.68E-07 & 6.33 & 0.101 & $\mathrm{G} / \mathrm{A}$ \\
\hline RBN & $122,501,344$ & 1 & $\mathrm{~V} 1, \mathrm{~V} 3$ & Chr4 & 8.91E-07 & 6.05 & 0.121 & $\mathrm{C} / \mathrm{T}$ \\
\hline RBN & $87,176,006$ & 1 & V1,V3 & Chr5 & 7.08E-07 & 6.15 & 0.123 & $\mathrm{C} / \mathrm{T}$ \\
\hline RBN & $221,805,144$ & 2 & V2 & Chr5 & $9.77 \mathrm{E}-07$ & 6.01 & 0.134 & $\mathrm{G} / \mathrm{A}$ \\
\hline RBN & $178,455,944$ & 2 & V2 & Chr5 & 2.29E-07 & 6.64 & 0.140 & $\mathrm{C} / \mathrm{T}$ \\
\hline RBN & $205,892,847$ & 2 & V2 & Chr5 & $2.82 \mathrm{E}-07$ & 6.55 & 0.122 & $\mathrm{~T} / \mathrm{C}$ \\
\hline RBN & $128,905,260$ & 1 & V2 & Chr6 & $8.32 \mathrm{E}-07$ & 6.08 & 0.114 & $\mathrm{G} / \mathrm{A}$ \\
\hline RBN & $162,388,475$ & 2 & V2 & Chr6 & $7.08 \mathrm{E}-07$ & 6.15 & 0.121 & $\mathrm{G} / \mathrm{A}$ \\
\hline RBN & $88,347,963$ & 1 & $\mathrm{~V} 1, \mathrm{~V} 3$ & Chr8 & 7.41E-07 & 6.13 & 0.138 & $\mathrm{~T} / \mathrm{C}$ \\
\hline RBN & $145,938,150$ & 2 & V2 & Chr9 & $5.25 \mathrm{E}-07$ & 6.28 & 0.150 & $\mathrm{G} / \mathrm{A}$ \\
\hline RBN, SUA & $58,218,452$ & 2 & $\mathrm{~V} 1, \mathrm{~V} 3$ & Chr7 & $1.12 \mathrm{E}-15$ & 14.95 & 0.302 & $A / G$ \\
\hline RBN,ROV, SUA & $43,293,834$ & 1 & $\mathrm{~V} 1, \mathrm{~V} 2$ & Chr2 & $1.29 \mathrm{E}-07$ & 6.89 & 0.110 & $\mathrm{G} / \mathrm{A}$ \\
\hline RDW & $227,871,089$ & 1 & V2 & Chr1 & 7.94E-07 & 6.10 & 0.140 & $\mathrm{C} / \mathrm{T}$ \\
\hline RDW & $82,567,249$ & 1 & V2 & Chr1 & 7.76E-07 & 6.11 & 0.140 & $C / G$ \\
\hline RDW & $2,246,081$ & 2 & V2 & Chr10 & $1.29 \mathrm{E}-11$ & 10.89 & 0.223 & $\mathrm{G} / \mathrm{A}$ \\
\hline RDW & $191,539,297$ & 2 & $\mathrm{~V} 1, \mathrm{~V} 3$ & Chr5 & 7.94E-07 & 6.10 & 0.160 & $\mathrm{G} / \mathrm{C}$ \\
\hline RDW & $82,882,718$ & 2 & V1,V3 & Chr5 & $1.35 \mathrm{E}-07$ & 6.87 & 0.063 & $C / A$ \\
\hline RDW & $118,512,703$ & 2 & V2 & Chr7 & $9.55 \mathrm{E}-07$ & 6.02 & 0.113 & $A / G$ \\
\hline RDW,SUA & $230,477,446$ & 1 & $\mathrm{~V} 1, \mathrm{~V} 3$ & Chr1 & 8.91E-07 & 6.05 & 0.051 & $\mathrm{G} / \mathrm{A}$ \\
\hline RDW/SDW & $19,943,384$ & 1 & V2 & Chr1 & 7.41E-09 & 8.13 & 0.160 & $\mathrm{C} / \mathrm{T}$ \\
\hline ROV & $227,871,089$ & 1 & $\mathrm{~V} 1, \mathrm{~V} 2, \mathrm{~V} 3$ & Chr1 & 8.71E-07 & 6.06 & 0.140 & $\mathrm{C} / \mathrm{T}$ \\
\hline ROV & $173,181,844$ & 1 & V2 & Chr1 & $6.46 \mathrm{E}-07$ & 6.19 & 0.120 & $\mathrm{G} / \mathrm{A}$ \\
\hline ROV & $150,754,726$ & 1 & V2 & Chr2 & $8.32 \mathrm{E}-07$ & 6.08 & 0.150 & $A / T$ \\
\hline ROV & $166,210,299$ & 1 & V2 & Chr2 & $5.01 \mathrm{E}-07$ & 6.30 & 0.122 & $\mathrm{C} / \mathrm{T}$ \\
\hline ROV & $21,486,113$ & 1 & V2 & Chr2 & 1.70E-07 & 6.77 & 0.110 & $\mathrm{C} / \mathrm{T}$ \\
\hline ROV & $187,822,582$ & 2 & V2 & Chr3 & $2.82 \mathrm{E}-12$ & 11.55 & 0.140 & $\mathrm{C} / \mathrm{T}$ \\
\hline ROV & $12,060,838$ & 2 & V2 & Chr3 & $1.00 \mathrm{E}-06$ & 6.00 & 0.100 & $\mathrm{~A} / \mathrm{G}$ \\
\hline ROV & $241,936,576$ & 1 & $\mathrm{~V} 1, \mathrm{~V} 2, \mathrm{~V} 3$ & Chr4 & 9.77E-07 & 6.01 & 0.052 & $C / G$ \\
\hline ROV & $118,806,068$ & 1 & V2 & Chr5 & $9.55 \mathrm{E}-07$ & 6.02 & 0.153 & $\mathrm{~T} / \mathrm{C}$ \\
\hline ROV & $28,955,506$ & 1 & V2 & Chr6 & $8.32 \mathrm{E}-07$ & 6.08 & 0.054 & $\mathrm{G} / \mathrm{A}$ \\
\hline ROV,SUA & $82,882,718$ & 2 & $\mathrm{~V} 1, \mathrm{~V} 2, \mathrm{~V} 3$ & Chr5 & 7.94E-15 & 14.10 & 0.228 & $C / A$ \\
\hline SUA & $227,871,089$ & 1 & V2 & Chr1 & $9.55 \mathrm{E}-08$ & 7.02 & 0.121 & $\mathrm{C} / \mathrm{T}$ \\
\hline SUA & $1,707,072$ & 1 & V2 & Chr2 & $3.55 \mathrm{E}-07$ & 6.45 & 0.200 & $\mathrm{G} / \mathrm{A}$ \\
\hline SUA & $2,610,094$ & 1 & V2 & Chr2 & 2.57E-08 & 7.59 & 0.121 & $C / G$ \\
\hline SUA & $94,781,431$ & 1 & $V_{2}, V_{3}$ & Chr3 & 7.94E-07 & 6.10 & 0.153 & $\mathrm{C} / \mathrm{T}$ \\
\hline
\end{tabular}


Table 6 Potential significant SNPs associated with root related traits (Continued)

\begin{tabular}{|c|c|c|c|c|c|c|c|c|}
\hline Traits & Position & Method $^{\mathrm{a}}$ & Stage & Chr & P-value & $-\log _{10}(P)$ & $\mathrm{R}^{2}$ & Genotype \\
\hline SUA & $7,141,374$ & 2 & $\mathrm{~V} 1, \mathrm{~V} 3$ & Chr5 & $6.46 \mathrm{E}-08$ & 7.19 & 0.152 & $\mathrm{G} / \mathrm{A}$ \\
\hline SUA & $217,144,020$ & 2 & V1,V3 & Chr5 & $1.55 \mathrm{E}-12$ & 11.81 & 0.160 & $\mathrm{~A} / \mathrm{T}$ \\
\hline SUA & $119,718,590$ & 1 & V2 & Chr5 & $5.50 \mathrm{E}-07$ & 6.26 & 0.150 & $C / T$ \\
\hline SUA & $179,029,112$ & 1 & V2 & Chr7 & 2.40E-07 & 6.62 & 0.145 & $C / T$ \\
\hline TRL & $72,599,769$ & 1 & $\mathrm{~V} 1, \mathrm{~V} 3$ & Chr1 & $4.68 \mathrm{E}-08$ & 7.33 & 0.100 & $\mathrm{~T} / \mathrm{C}$ \\
\hline TRL & $111,734,317$ & 2 & V2 & Chr2 & $7.08 \mathrm{E}-07$ & 6.15 & 0.128 & $A / G$ \\
\hline TRL & $59,237,040$ & 1 & V2 & Chr3 & 5.37E-08 & 7.27 & 0.134 & $\mathrm{G} / \mathrm{A}$ \\
\hline TRL & $241,936,576$ & 1 & $\mathrm{~V} 1, \mathrm{~V} 3$ & Chr4 & $2.95 \mathrm{E}-07$ & 6.53 & 0.061 & $C / G$ \\
\hline TRL & $128,905,254$ & 1 & V2 & Chr6 & $5.13 \mathrm{E}-07$ & 6.29 & 0.106 & $\mathrm{G} / \mathrm{A}$ \\
\hline
\end{tabular}

MAF minor allele frequency, $R^{2}$ phenotypic contribution, Chr chromosome, $R D W$ root dry weight, $R D W / S D W$ root per shoot dry weight, TRL total root length, SUA surface area, $A R D$ average root diameter, $R O V$ root volume, $R B N$ root branching number

a method 1-3 refers to EMMAX, FarmCPU, and MLM, respectively

\section{Expression levels analysis}

Five candidate genes including Zm00001d015379, Zm00001d050783, Zm00001d018496, Zm00001d038676, and Zm00001d017751 harboring significant SNPs (with phenotypic contribution rates greater than $12 \%$ ) within exonic regions were tested for expression levels using maize accessions with extreme root branching number differences from both GWAS and mapping panels at two growth stages (V1, V3). The relative expression level results obtained through qRT-PCR revealed that three candidate genes viz. Zm00001d038676, Zm00001d015379, and Zm00001d018496 acted as positive regulators for root branching number while two genes viz. Zm00001d050783 and $\mathrm{Zm00001d017751} \mathrm{acted} \mathrm{as} \mathrm{negative} \mathrm{regulators} \mathrm{for} \mathrm{root}$ branching in both GWAS and mapping accessions (Figs. 5 and 6) at all the considered stages (V1, V3). The expression

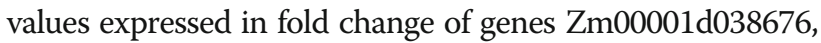
Zm00001d015379, and Zm00001d018496 in both GWAS and mapping accessions with high root branching number were significantly higher $(P<0.05, P<0.01, P<0.001)$ than those of accessions with low root branching number at all

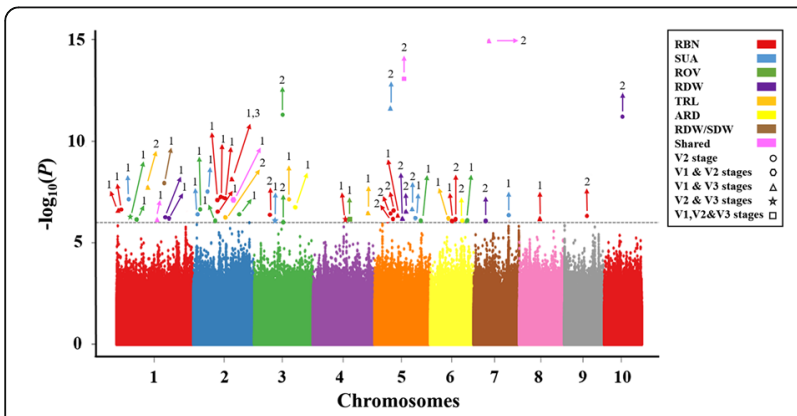

Fig. 4 Manhattan plot of all potential significant SNPS associated with each root-related trait at each specific stage (V1, V2, and V3). 1, 2, 3 refer to EMMAX, FarmCPU, and MLM, respectively. RDW = root dry weight; RDW/SDW = root per shoot dry weight; $\mathrm{TRL}=$ total root length; SUA = surface area; $\mathrm{ARD}=$ average root diameter; $\mathrm{ROV}=$ root volume; $\mathrm{RBN}=$ root branching number
V1 and V3 stages (Figs. 5 and 6). In contrast, genes Zm00001d050783 and Zm00001d017751 displayed significantly lower expression levels in accessions with high root branching number as compared to low root branching number accessions (Figs. 5 and 6).

\section{Discussion}

The use of natural variation approaches not only promotes the genetic basis of complex traits and the discovery of new regulators but also, can facilitate the identification of interesting alleles that can be further used to dissect the molecular mechanisms of the genes underlying the trait variation, which may be directly used for breeding purposes [4]. In this study, wide ranges of variations in terms of seedling root related traits were observed at both stages investigated. In regards to all traits, root length and branching number showed the largest phenotypic variations. Thus far, considerable natural phenotypic variations for root system architecture traits in various maize panels have been reported [2, 24, 31, 44, 46, 47]. Broad sense heritability estimates were relatively high with similar tendencies across stages. Recently, inline heritability ranges have been recorded in similar studies regarding maize seedling root traits at various growth stages/time-points both under field and controlled conditions $[8,15]$. The traits like root volume, total root length, surface area, and root branching number were found tightly correlated in this current investigation. Positive significant associations between seedling and adult root traits were earlier reported by Abdel-Ghani et al. [17]. These findings were consistent with those observed by several investigators [2, 24, 44]. Root dry weight, surface area and total root length were stated to affect plant's nutrients and water assimilation and absorption [48].

Most of the earlier root-related studies have been carried out using low-density genetic linkage maps involved commonly simple sequence repeat (SSR) or restriction 
Table 7 Candidate genes associated with potential SNPs and functional annotations

\begin{tabular}{|c|c|c|c|c|c|c|}
\hline Gene_ID & Traits & Chr & $\begin{array}{l}\text { SNP } \\
\text { Position }\end{array}$ & Distance(bp) & Gene type & Functional annotation/biological pathway \\
\hline Zm00001d043773 & ARD & 3 & $\begin{array}{l}209,661 \\
144\end{array}$ & 0 & $\begin{array}{l}\text { exon, } \\
\text { synonymous }\end{array}$ & Putative clathrin assembly protein \\
\hline Zm00001d038558 & ARD & 6 & $\begin{array}{l}159,805 \\
368\end{array}$ & 0 & UTR3 & cystatin3, CC3, Cysteine proteinase inhibitor 2, \\
\hline Zm00001d029482 & RBN & 1 & $\begin{array}{l}72,599 \\
741\end{array}$ & 0 & intronic & NAD(P)-binding Rossmann-fold superfamily protein \\
\hline Zm00001d030376 & RBN & 1 & $\begin{array}{l}127,352 \\
056\end{array}$ & 0 & intronic & ATP-dependent DNA helicase \\
\hline Zm00001d001900 & RBN & 2 & $2,564,296$ & 0 & intronic & Probable cysteine protease RD21B \\
\hline Zm00001d003119 & RBN & 2 & $\begin{array}{l}32,824 \\
965\end{array}$ & 0 & intronic & $\begin{array}{l}\text { Trafficking protein particle complex II-specific subunit } 120 \\
\text { homolog }\end{array}$ \\
\hline Zm00001d005925 & RBN & 2 & $\begin{array}{l}192,996 \\
724\end{array}$ & 0 & UTR5 & Glucose-6-phosphate isomerase 1 chloroplastic, \\
\hline Zm00001d006030 & RBN & 2 & $\begin{array}{l}\text { 195,707, } \\
091\end{array}$ & 0 & intergenic & ENT domain-containing protein \\
\hline Zm00001d042535 & RBN & 3 & $\begin{array}{l}171,057 \\
172\end{array}$ & 0 & intronic & selenoprotein family protein \\
\hline Zm00001d050783 & RBN & 4 & $\begin{array}{l}122,501 \\
344\end{array}$ & 0 & $\begin{array}{l}\text { exon, } \\
\text { nonsynonymous }\end{array}$ & Molybdopterin synthase sulfur carrier subunit \\
\hline Zm00001d015379 & RBN & 5 & $\begin{array}{l}87,176 \\
006\end{array}$ & 0 & $\begin{array}{l}\text { exon, } \\
\text { nonsynonymous }\end{array}$ & Splicing factor arginine/serine-rich 12 \\
\hline Zm00001d016858 & RBN & 5 & $\begin{array}{l}178,455 \\
944\end{array}$ & 0 & UTR3 & Ankyrin repeat protein SKIP35 \\
\hline Zm00001d017751 & RBN & 5 & $\begin{array}{l}205,892 \\
847\end{array}$ & 0 & $\begin{array}{l}\text { exon, } \\
\text { nonsynonymous }\end{array}$ & Pentatricopeptide repeat-containing protein (chloroplastic) \\
\hline Zm00001d018496 & RBN & 5 & $\begin{array}{l}221,805 \\
144\end{array}$ & 0 & $\begin{array}{l}\text { exon, } \\
\text { synonymous }\end{array}$ & Pumilio homolog 4 \\
\hline Zm00001d037546 & RBN & 6 & $\begin{array}{l}128,905 \\
260\end{array}$ & 0 & intronic & unknown \\
\hline Zm00001d038676 & RBN & 6 & $\begin{array}{l}162,388 \\
475\end{array}$ & 0 & $\begin{array}{l}\text { exon, } \\
\text { synonymous }\end{array}$ & Probable xyloglucan glycosyltransferase 12 \\
\hline Zm00001d009896 & RBN & 8 & $\begin{array}{l}88,347 \\
963\end{array}$ & 0 & $\begin{array}{l}\text { exon, } \\
\text { nonsynonymous }\end{array}$ & unknown \\
\hline Zm00001d047946 & RBN & 9 & $\begin{array}{l}145,938 \\
150\end{array}$ & 0 & intronic & Cell cycle checkpoint protein RAD17 \\
\hline Zm00001d003405 & $\begin{array}{l}\text { RBN, ROV, } \\
\text { SUA }\end{array}$ & 2 & $\begin{array}{l}43,293 \\
834\end{array}$ & 5823 & intergenic & $\begin{array}{l}\text { Bifunctional inhibitor/lipid-transfer protein/seed storage } 2 \mathrm{~S} \\
\text { albumin superfamily protein }\end{array}$ \\
\hline Zm00001d001841 & RBN, SUA & 2 & $1,707,072$ & 0 & intronic & unknown \\
\hline Zm00001d019766 & RBN, SUA & 7 & $\begin{array}{l}58,218 \\
452\end{array}$ & -5544 & intergenic & RING/U-box superfamily protein \\
\hline Zm00001d029683 & RDW & 1 & $\begin{array}{l}82,567 \\
249\end{array}$ & 0 & $\begin{array}{l}\text { exon, } \\
\text { nonsynonymous }\end{array}$ & irregular pollen exine1 \\
\hline Zm00001d017279 & RDW & 5 & $\begin{array}{l}191,539 \\
297\end{array}$ & 0 & $\begin{array}{l}\text { exon, } \\
\text { nonsynonymous }\end{array}$ & Phenylalanine ammonia lyase7 \\
\hline Zm00001d020485 & RDW & 7 & $\begin{array}{l}118,512 \\
703\end{array}$ & 0 & UTR5 & Golgi SNAP receptor complex member 1 \\
\hline Zm00001d023292 & RDW & 10 & $2,246,081$ & 0 & $\begin{array}{l}\text { exon, } \\
\text { synonymous }\end{array}$ & Trigger factor-like protein TIG Chloroplastic \\
\hline Zm00001d015290 & $\begin{array}{l}\text { RDW, ROV, } \\
\text { SUA }\end{array}$ & 5 & $\begin{array}{l}82,882 \\
718\end{array}$ & 2998 & intergenic & Adagio protein 1 \\
\hline Zm00001d032558 & RDW, SUA & 1 & $\begin{array}{l}230,477 \\
446\end{array}$ & 20,619 & intergenic & unknown \\
\hline
\end{tabular}


Table 7 Candidate genes associated with potential SNPs and functional annotations (Continued)

\begin{tabular}{|c|c|c|c|c|c|c|}
\hline Gene_ID & Traits & Chr & $\begin{array}{l}\text { SNP } \\
\text { Position }\end{array}$ & Distance(bp) & Gene type & Functional annotation/biological pathway \\
\hline Zm00001d028001 & RDW/SDW & 1 & $\begin{array}{l}19,943 \\
384\end{array}$ & 0 & UTR3 & Unknown \\
\hline Zm00001d031009 & ROV & 1 & $\begin{array}{l}173,181 \\
844\end{array}$ & 0 & intronic & Protein tesmin/TSO1-like CXC 2 \\
\hline Zm00001d002751 & ROV & 2 & $\begin{array}{l}21,486 \\
113\end{array}$ & 0 & intronic & Probable isoaspartyl peptidase/L-asparaginase 3 \\
\hline Zm00001d004960 & ROV & 2 & $\begin{array}{l}150,754 \\
726\end{array}$ & 0 & intronic & 2-isopropylmalate synthase 1 chloroplastic \\
\hline Zm00001d005264 & ROV & 2 & $\begin{array}{l}166,210 \\
299\end{array}$ & 0 & $\begin{array}{l}\text { exon, } \\
\text { synonymous }\end{array}$ & Tetratricopeptide repeat (TPR)-like superfamily protein \\
\hline Zm00001d039693 & ROV & 3 & $\begin{array}{l}12,060 \\
838\end{array}$ & 0 & intronic & Protein RST1 \\
\hline Zm00001d043059 & ROV & 3 & $\begin{array}{l}187,822 \\
582\end{array}$ & 0 & UTR5 & Protein FATTY ACID EXPORT 3 chloroplastic, \\
\hline Zm00001d015779 & ROV & 5 & $\begin{array}{l}118,806 \\
068\end{array}$ & 0 & intronic & 14-3-3-like protein GF14 omega \\
\hline Zm00001d035487 & ROV & 6 & $\begin{array}{l}28,955 \\
506\end{array}$ & 0 & intronic & E3 SUMO-protein ligase SIZ1 \\
\hline Zm00001d032473 & $\begin{array}{l}\text { ROV, RDW, } \\
\text { SUA }\end{array}$ & 1 & $\begin{array}{l}227,871 \\
089\end{array}$ & 0 & $\begin{array}{l}\text { exon, } \\
\text { nonsynonymous }\end{array}$ & CDPK-related kinase 3 \\
\hline Zm00001d053827 & ROV, TRL & 4 & $\begin{array}{l}241,936 \\
576\end{array}$ & -2034 & intergenic & BEACH domain-containing protein B \\
\hline Zm00001d001901 & SUA & 2 & $2,610,094$ & 0 & UTR3 & Reticulon-like protein B11 \\
\hline Zm00001d041070 & SUA & 3 & $\begin{array}{l}94,781 \\
431\end{array}$ & 0 & intronic & 5-methylthioadenosine/S-adenosylhomocysteine deaminase, \\
\hline Zm00001d013252 & SUA & 5 & $7,141,374$ & 0 & UTR3 & $60 \mathrm{~S}$ ribosomal protein L13a-1 \\
\hline Zm00001d015788 & SUA & 5 & $\begin{array}{l}119,718 \\
590\end{array}$ & 0 & UTR3 & proteasome component $4,26 \mathrm{~S}$ protease regulatory subunit S10B \\
\hline Zm00001d018235 & SUA & 5 & $\begin{array}{l}217,144 \\
020\end{array}$ & 0 & intronic & unknown \\
\hline Zm00001d022502 & SUA & 7 & $\begin{array}{l}179,029 \\
112\end{array}$ & 0 & intronic & Exocyst complex component Sec. 8 \\
\hline Zm00001d029482 & TRL & 1 & $\begin{array}{l}72,599 \\
769\end{array}$ & 0 & intronic & NAD(P)-binding Rossmann-fold superfamily protein \\
\hline Zm00001d004438 & TRL & 2 & $\begin{array}{l}111,734 \\
317\end{array}$ & 0 & intronic & Pullulanase-type starch debranching enzyme1 \\
\hline Zm00001d040704 & TRL & 3 & $\begin{array}{l}59,237 \\
040\end{array}$ & 0 & $\begin{array}{l}\text { exon, } \\
\text { nonsynonymous }\end{array}$ & ATP-dependent DNA helicase \\
\hline Zm00001d037546 & TRL & 6 & $\begin{array}{l}128,905 \\
254\end{array}$ & 0 & intronic & unknown \\
\hline
\end{tabular}

$R D W$ root dry weight, $R D W / S D W$ root per shoot dry weight, TRL total root length, SUA surface area, $A R D$ average root diameter, $R O V$ root volume, $R B N$ root branching number, Chr chromosome

fragment length polymorphism (RFLP), resulting in scanty inter-marker intervals not efficient enough for accurate QTL and gene identification [49]. In crops, association studies based on LD were previously stated as an effective approach for detecting and identifying SNPs or genes correlated with complex traits, such as roots [50]. GWAS use millions of single nucleotide polymorphisms (SNPs) markers to determine alleles associated with multiple traits in crops and identify genes controlling their expression [51]. In this analysis, a total of 1,490,007 consistent SNP markers were distributed among the ten maize chromosomes to directly mine SNPs and genes putatively associated with seven root architectural traits viz. RDW, RDW/SDW, TRL, SUA, ARD, ROV, and RBN across three vegetative stages (V1, V2, and V3) and using three GWAS models (MLM, EMMAX, and FarmCPU). Abundant polymorphisms and fast LD decay make maize an excellent crop for association studies 


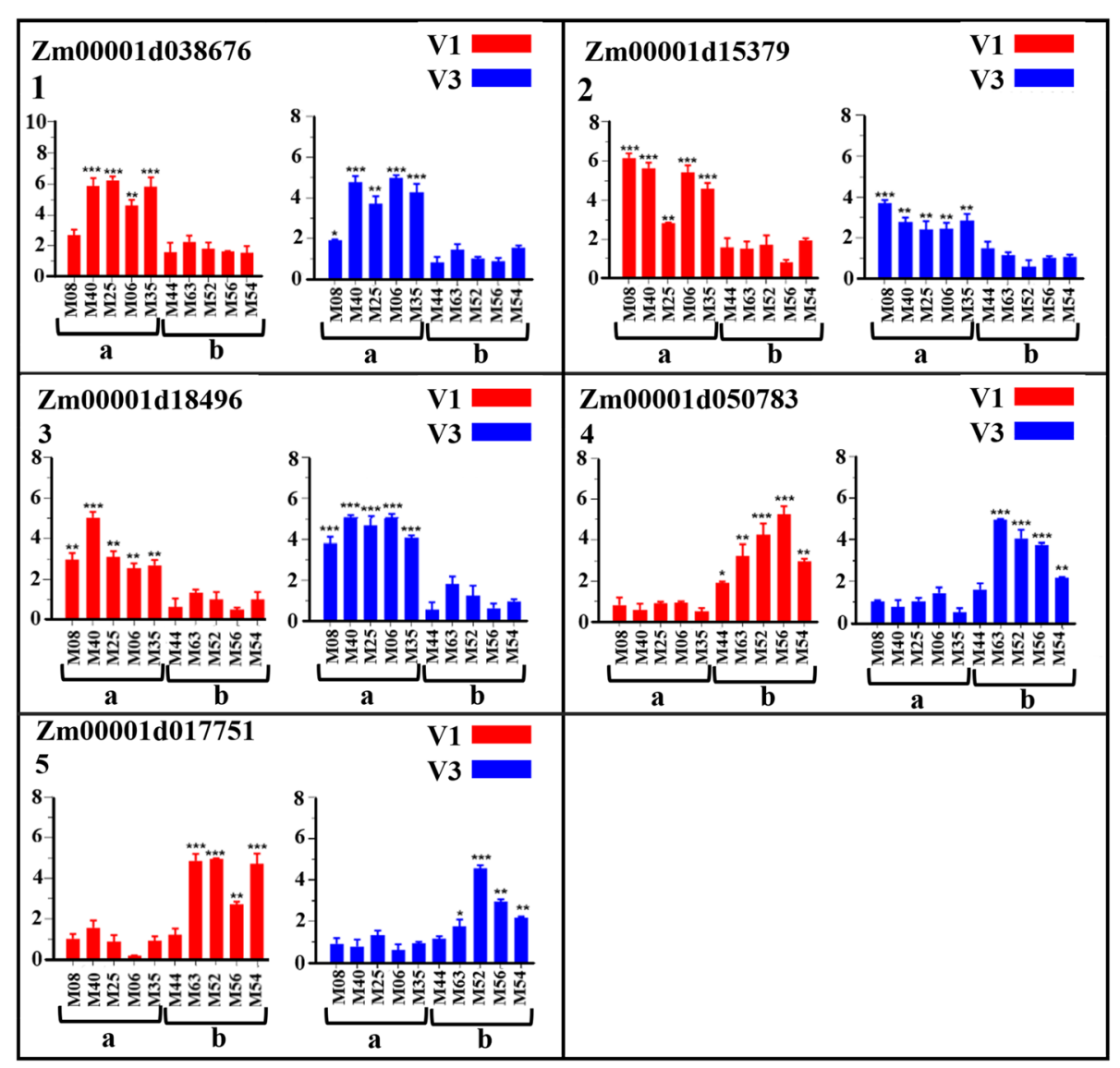

Fig. 5 Relative expression levels (mean from three replicates) of five putative candidate genes (1 to 5) at V1 (in red bars) and V3 (in blue bars) growth stages in phenotypically extreme maize accessions for root branching number trait from the GWAS panel. Values of fold difference are shown in mean \pm standard deviation (error bar). Relative expression levels were calculated using the $2 \wedge(-\Delta \Delta c t)$ method. 1,2 , and 3 are positive regulating genes while 4 and 5 are negative regulating genes. $a$ and $b$ stand for high and low root branching number accessions, respectively. ***, **, and * indicate the significance level for $P<0.001, P<0.01$ and $P<0.05$, respectively

[52]. The average LD decay distance was $5.2 \mathrm{~kb}$, which specially enhances SNPs and genes mapping accuracy as compared to the reported densities for GBS-SNP markers ranging from $6.2 \mathrm{~kb}$ to $100 \mathrm{Mbp}[8,44]$ in recent maize root-trait QTL studies (last summary) [22]. These results are consistent with the reported average LD decay for inbred maize lines occurring within 1$10 \mathrm{~kb}[2,53]$. Based on significant SNPs (for multiple testing critical threshold of $\left.-\log _{10}(\mathrm{P}) \geq 6.0\right)$ identified within candidate genes or across at least two stages/ methods simultaneously, 53 potential SNPs were detected. Comparatively, EMMAX and FarmCPU were the most efficient and accurate models for detecting 34 SNPs and 19 SNPs, respectively, while MLM model was the least efficient by detecting only one significant SNP. Due to its high stringency, MLM was earlier noted to create type II errors and cause false negatives [2, 44]. Recently, in a similar study, Sanchez et al. monitored fourteen SNPs through FarmCPU and four SNPs by MLM using a panel of 62,077 SNP markers [44]. It was previously indicated that various algorithmic approaches ought to be used to perform GWAS analysis in a real application for complex trait studies due to the limitation in a single model to detect polygenic variation associations [54-56]. A large number of root-related trait SNPs detected in this study are consistent with known genes recorded in previous findings. Using the results of fifteen root QTL studies from nine different bi-parental mapping populations, a meta-analysis study of QTLs recapitulated many putative MQTLs related to maize root development [57]. Interestingly, our detected SNPs were found to be in LD with four noteworthy loci involved in root development throughout developmental stages, namely S1_227871089 with Ax-2 (at bin 1.07), S2 32824965 and S2_43293834 with Rt-6 (at bin 2.04), S3 171057172, and S3_187822582 with Rt-7 (at bin 3.06), and S6_128905254 and S6_128905260 with Rt-13 (at bin 6.05) [57]. SNP S1_227871089 on chromosome 1 significantly associated with ROV, RDW and SUA housed

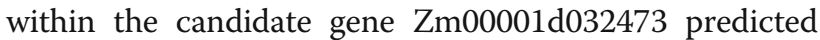




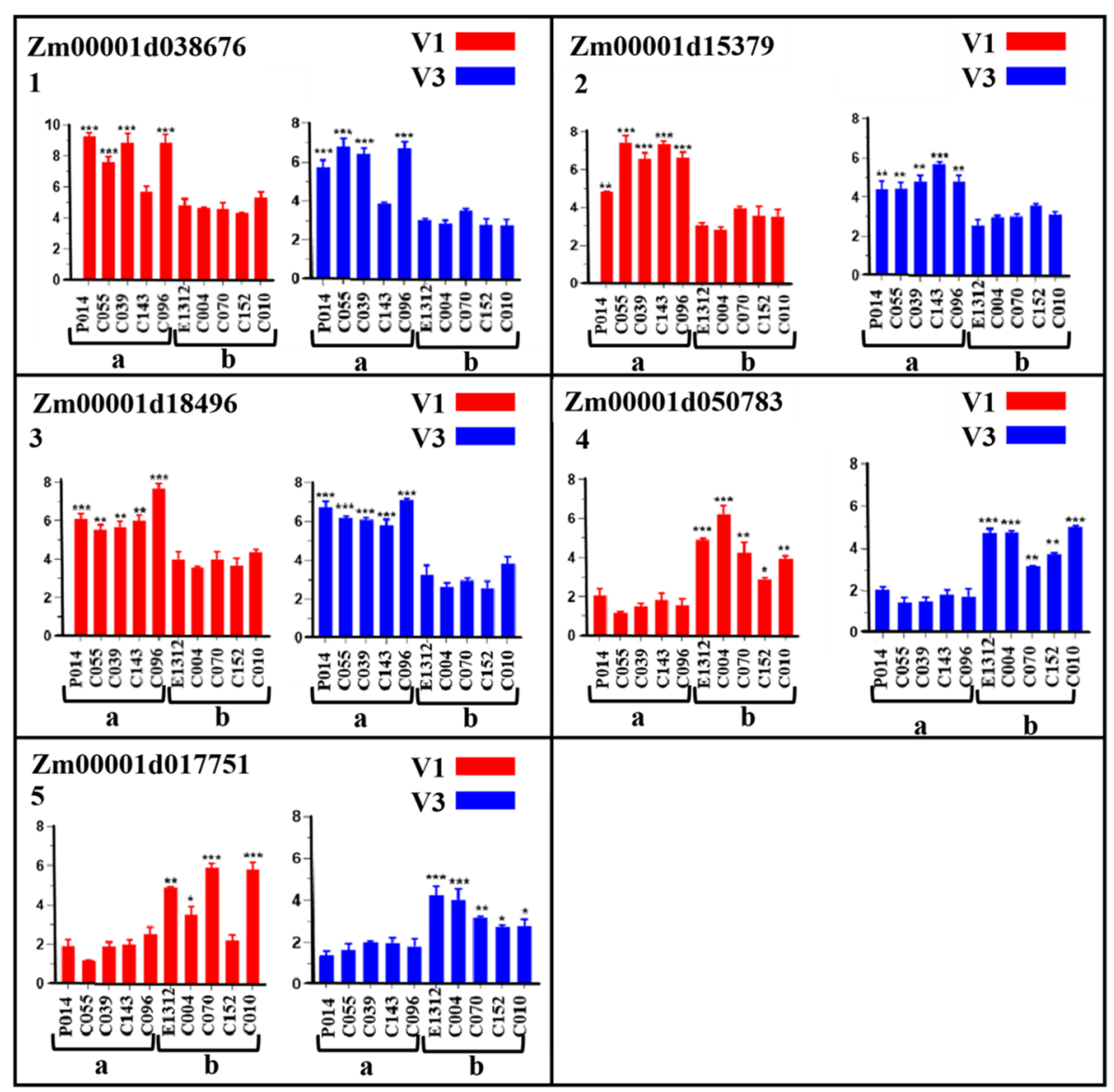

Fig. 6 Relative expression levels (mean from three replicates) of five putative candidate genes (1 to 5) at V1 (in red bars) and V3 (in blue bars) growth stages in phenotypically extreme maize accessions for root branching number trait from the biparental population. Values of fold difference are shown in mean \pm standard deviation (error bar). Relative expression levels were calculated using the $2 \wedge(-\Delta \Delta \mathrm{ct})$ method. 1,2 , and 3 are positive regulating genes while 4 and 5 are negative regulating genes. a and $b$ stand for high and low root branching number accessions, respectively. ${ }^{* * *}{ }^{* *}$, and ${ }^{*}$ indicate the significance level for $P<0.001 ; P<0.01$ and $P<0.05$, respectively

to encode a CDPK-related kinase 3 enzyme highly expressed in primary root growth [58-60]. This SNP was also in LD with the detected cluster QTLs qRDW $\mathrm{v3}^{-}$ 1-1 and qRDW/SDW $\mathrm{v3}-1-1$ in addition to qTRSA21-1, qTRL21-1 [19], and ${ }_{9 d} R T N 1-1$ [24]. SNP S4-122501344 associated with RBN was located within gene Zm00001d050783 which codes molybdopterin synthase sulfur carrier subunit. This SNP was also in LD with the detected QTLS $\mathrm{qSUA}_{\mathrm{v} 1}-4-1$, $\mathrm{qSUA}_{\mathrm{v} 2}-4-1$, and $\mathrm{qROV}_{\mathrm{v} 2}$ 4-1 in addition to the gene model GRMZM2G32186 [2] associated with several SNPs for root length, which showed high expression in the maize primary root at emergence and V1 stage [61]. Furthermore, SNP S4122501344 was found to be within another reported gene (GRMZM2G153722) with high expression in seedling root and shoot [61] which contains nine other SNPs associated with root diameter and surface area [2]. SNPs S5_87176006 and S5_205892847 associated with RBN were located within gene models $\mathrm{Zm00001d015379}$ and
Zm00001d017751 which encode for an arginine/serinerich protein 12 and a pentatricopeptide repeatcontaining protein At2g15820 like, respectively. These SNPs were in LD with a QTL for crown root length in bin 5.04 detected by Liu et al. [15]. SNPs S6_128905254 and S6_128905260 significantly associated with TRL and $\mathrm{RBN}$ were found to be within the gene model Zm00001d037546 with currently unknown function. Its associated synonymous gene (GRMZM2G030235) highly expressed in maize seedling primary root at both VE and V1 stages [58, 61]. These SNPs were furthermore in LD with qARL26-1, qARN26-1 [19] and a QTL for seminal root number in bin 6.05 reported by Liu et al. [15]. SNP S7_118512703 associated with RDW on chromosome 7 was found within Zm00001d020485, a gene encoding a Golgi SNAP receptor complex member 1. This SNP was also in LD with $q T R L 17-1$ [19] and ${ }_{9 d} L R L 7-1$ [24]. Remarkably, the cluster $\mathrm{qTRL}_{\mathrm{v} 1}-10-1$ and $\mathrm{qRBN} \mathrm{v1}_{1}-10-1$ associated with total root length and root branching 
number at V1 stage collocated consistently with the QTL $q T R L_{5 d^{-10-1}}$ regulating total root length detected recently by Moussa et al. [45] within the same population using 5 days old seedlings. This chromosomal region can, therefore, be considered as a special noteworthy locus that can directly be used in markerassisted selection programs.

When looking for SNPs within identified mutants or cloned genes for maize root development, rth6 [62] was putatively in LD with SNPs S1_127352056 and S1_ 173181844 associated with RBN and ROV; rth5 [63] was in LD with SNPs S3_171057172 and S3_187822582 associated with RBN and ROV; rum1 [64, 65] was in LD with SNP S3_209661144 associated with ARD; and rth2 [66] was in LD with SNPs S5_87176006, S5_118806068, S5_119718590, S5_205892847, and S5_82882718 associated with RBN, ROV, SUA, and RDW.

Gene expression profile can show whether the gene possesses a biological function [67]. However, several genes have been broadly determined through GWAS, but most of them are new genes without functional verification. Today, qRT-PCR is widely used to validate GWAS-detected gene expression with high accuracy and sensitivity $[68,69]$. In this study, five potential genes were checked for expression levels using maize accessions with extreme root branching number differences. At all considered growth stages (V1, V3), three genes viz. Zm00001d038676, Zm00001d015379, and Zm00001d018496 acted as positive regulators for root branching number with significantly higher expression levels in high root branching number maize accessions. Conversely, two other genes viz. Zm00001d050783 and Zm00001d017751 with significantly lower expression in high root branching number accessions were found to act as negative regulating genes. The identified SNPs and evaluated genes by enriching our insights into the molecular mechanisms of maize root architecture, could thus be of great value for future marker-assisted breeding programs. Upcoming research will, however thoroughly elucidate the function of these genes in maize root growth and development.

\section{Conclusions}

This study provides a comprehensive analysis of the genetic architecture of root traits in elite maize lines. A recombinant inbred line population and a genome-wide association study panel were used for mapping loci associated with maize root architectural traits at three developmental stages under standard conditions. QTL mapping using inclusive composite interval mapping identified eight QTLs for root traits on chromosomes $1\left(\mathrm{qRDW}_{\mathrm{v} 3}-1-1\right.$ and $\left.\mathrm{qRDW} / \mathrm{SDW}_{\mathrm{v} 3}-1-1\right), 2\left(\mathrm{qRBN}_{\mathrm{v} 1}-2-1\right)$, 4. $\left(\mathrm{qSUA}_{\mathrm{v} 1}-4-1\right.$, $\mathrm{qSUA}_{\mathrm{v} 2}-4-1$, and $\left.\mathrm{qROV}_{\mathrm{v} 2}-4-1\right)$, and 10 $\left(\mathrm{qTRL}_{\mathrm{v} 1}-10-1, \mathrm{qRBN}_{\mathrm{v1}}-10-1\right)$, and each QTL explained
$6.44-8.83 \%$ of phenotypic variation. Genome-wide association study analysis identified 53 substantial SNPs through three GWAS models (EMMAX, FarmCPU, and MLM). The detected SNPs showed individual phenotypic contribution rates ranging from 5.10 to $30.2 \%$ and were significantly associated with RBN (17), ROV (10), SUA (8), RDW (6), TRL (5), ARD (2), and RDW/SDW (1). Within the LD region of $5.2 \mathrm{~kb}$ for the significant SNPs, 46 candidate genes were identified. Five promising genes viz. Zm00001d038676, Zm00001d015379, Zm00001d018496,

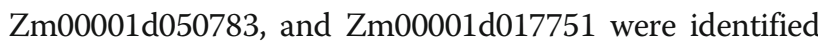
and successfully verified for expression level. The evaluated genes were shown to serve as positive/negative regulators for root branching in the spring maize lines. Thus, SNPs and underlying genes discovered in the present study could be critical for future marker-assisted breeding programs of high-efficient root systems in maize, as well as supporting the breeding of high-yielding maize varieties.

\section{Methods \\ Plant materials, growth chamber experiment, and traits measurement}

In this study, two genetic populations were used. The GWAS panel comprised 80 natural maize inbred lines covering more than $80 \%$ planting area in Jilin Province (China). All the accessions are the same as defined in our earlier study [52]. The mapping population consisted of 179 RILs developed by a cross between the female parent P014 and the male parent E1312 and continuous inbreeding for 9 generations. P014 line was known to possess a larger and thicker root system with significantly higher root dry weight, total root length, surface area, projected area, average root diameter, and root tips as compared to E1312 line [45]. Genetically pure seeds from both populations were produced at the Jilin Agricultural University's experimental field in summer 2019. A controlled growth chamber experiment was conducted using a completely randomized design (CRD) with three replications. The growth chamber parameters were: 28/ $25^{\circ} \mathrm{C}$ temperature, $14 / 10 \mathrm{~h}$ (light/darkness) photoperiod, and $70 / 80 \%$ relative humidity day/night, respectively. The light intensity was set at $200 \mu \mathrm{mol}$ photons $\mathrm{m}^{-2} \mathrm{~s}^{-1}$. The seeds from both GWAS and mapping populations were directly planted in polyvinyl chloride (PVC) pipes $(8 \mathrm{~cm}$ bottom diameter, $3.2 \mathrm{~mm}$ thickness, and $25 \mathrm{~cm}$ height) containing a mixture of sandy soil and vermiculite (2:1 ratio). Root architectural traits were investigated at three vegetative stages of maize growth namely V1 (one fully expanded leaf), V2 (two fully developed leaves), and V3 (three fully developed leaves) which approximately corresponded to 5,15 and 25 days after emergence, respectively. For data collection, each PVC pipe consisted of one seedling was considered an 
experimental unit and the growth chamber independent trials were completed in February, April, and July 2020. All the experiments were repeated three times to increase the reliability of root quantitative traits measurements. The seedlings were cautiously removed from soil at each specific stage and washed with running water to eliminate the soil residues. For each seedling, the root system was separated from the shoot, and roots were scanned using a root scanner-based image (Perfection V800 Epson, resolution of 12,800 dots per inch (dpi: 5039.37 dots per $\mathrm{cm}$ )) then analyzed via DJ-GXG02 software [70]. If the collection of data could not be carried out within one single day, seedlings were kept in $30 \%$ ethanol by swamping the roots and then placed in a cold chamber $\left(4^{\circ} \mathrm{C}\right)$ with reference to Sanchez et al. [44]. A total of seven root related traits namely root dry weight, root to shoot dry weight, total root length, surface area, root volume, average root diameter, and root branching number were collected (Table 8). For dry weight biomass, root and shoot were recorded individually using an electronic weighing balance once drying in an oven set at $75^{\circ} \mathrm{C}$ for at least $48 \mathrm{~h}$ to achieve the constant weight.

\section{Phenotypic analysis of root related traits}

The descriptive analyses were carried out using Minitab17 program (Minitab Inc., State College, PA, USA). For every root related trait at each stage and in both populations, descriptive statistics including mean, standard deviation, maximum, minimum, skewness, kurtosis, and coefficient of variation were calculated. The broadsense heritability $\left(\mathrm{H}^{2}\right)$ was determined using variance components obtained via ANOVA as described by Pace et al. [2]. Normal distribution and Pearson coefficients of correlation among traits were also generated. Figures were produced using GraphPad Prism 8.0.1 (GraphPad Software, Inc., San Diego, CA).

Genotyping, linkage map construction, and QTL mapping The 179 RILs were genotyped by sequencing (GBS), and MSTmap was used for linkage analysis of marker data [71]. Linkage map was constructed based on automatic parameter settings with reference to Meng et al. [72]. Briefly, the markers were grouped at a LOD of $\geq 3.0$, ordered, rippled, and then outputted to generate the linkage map. QTL IciMapping 4.1 software was used to perform QTL analysis using inclusive composite interval mapping for additive QTL (ICIM-ADD) [73]. The walking speed was $1.0 \mathrm{cM}$ and the size of the windows was $5.0 \mathrm{cM}$. A LOD threshold peak score value of $\geq 2.5$, which is commonly used in maize QTL mapping [49, 74], was set to declare significant QTLs. QTL additive effects and phenotypic variance explained (PVE) were also analysed.

Next-generation sequencing of GWAS population genome Genomic DNA was extracted from leaves for the 80 maize inbred lines using a cetyltrimethylammonium bromide (CTAB) protocol for plant tissues [75]. The different accessions were genotyped via next-generation sequencing at Novogene Biological Company (https:// novogene.com/, Beijing, China). Briefly, for genomic libraries construction, the DNA samples were digested by sonication to a size of $350 \mathrm{bp}$, the genomics fragments were then subjected to end polishing and A-tailing afterward ligated to the full-length adapter for Illumina HiSeq PE150 platform (Illumina Inc., San Diego, CA, USA). Subsequently, the libraries were analyzed using Agilent 2100 Bioanalyzer and high-consistent sequencing data that show polymorphisms were then mapped to the B73 maize reference genome (RefGen_v3) using BWA software [76]. Duplicates were expurgated using SAMtoots [77]. Thus, 34,872,961 SNPs were obtained from next-generation sequencing analysis. Finally, using the Bayesian model mpileup of SAMtools with the criteria of SNP missing rate of $<10 \%$ and minor allele frequency (MAF) of $\geq 5 \%$, a final total of $1,490,007$ high-consistent SNP markers were obtained for genetic evolution and GWAS analyses.

\section{Population structure and linkage disequilibrium analysis} Based on a total of 1,490,007 high-consistent SNP markers, the principal component analysis (PCA) of individuals was performed using genome-wide complex

Table 8 Collected root related traits initials and illustrations

\begin{tabular}{lll}
\hline Trait Name & Abbreviations & Trait description \\
\hline Root dry weight & RDW & Total root dry weight of the seedling in gram \\
Root to shoot dry weight & RDW/SDW & Root to shoot dry weight ratio in gram \\
Total root length & TRL & Cumulative length of the root system in $\mathrm{cm}$ \\
Surface area & SUA & Whole root system surface area in $\mathrm{cm}^{2}$ \\
Root volume & ROV & Cumulative volume of all the roots in $\mathrm{cm}^{3}$ \\
Average root diameter & ARD & Average diameter of the entire root system in $\mathrm{mm}$ \\
Root branching number & RBN & Total number of all the root tips \\
\hline
\end{tabular}


trait analysis software tool (GCTA) [78]. The distances between the materials were inferred based on the distance matrix for phylogenetic tree contruction using TreeBeST program (http://treesoft.sourceforge.net/ treebest.shtml/). Using PLINK [79], the decay of linkage disequilibrium measured in base pairs was calculated on each chromosome using an $\mathrm{r}^{2}$ value of 0.1 as a cut-off.

\section{GWAS analysis}

In this study, to control false positive or spurious associations, three GWAS models were implemented, viz.: (1) Mixed Linear Model (MLM) [80], where PCA (Q) from population structure and kinship (K) were used as covariates; (2) Efficient Mixed-Model Association eXpedited (EMMAX), which outperforms both PCA and genomic control in correcting for sample structure with high statistical power [81] and; (3) Fixed and random model Circulating Probability Unification (FarmCPU), where PCA (as a fixed effect) and kinship (as a random effect) were used as covariates [82]. GWAS using the MLM model and EMMAX model was performed using the software TASSEL 5.0 [83], and FarmCPU method was performed using $\mathrm{R}$ FarmCPU package [82]. The threshold was defined based on the number of effective SNP markers. Multiple testing using simpleM program integrated in R, which calculates the number of informative SNP markers (Meff_G) was used to set the threshold. Briefly, a correlation matrix for all 1,490,007 SNPs was generated and the corresponding eigenvalues were calculated; a composite LD correlation was then calculated directly using GAPIT package and once this SNP matrix was obtained, Meff_G was calculated and this value was used to compute for the multiple testing threshold in the same way as the Bonferroni correction method, where the significance threshold $(\alpha=0.05)$ was divided by the Meff_G $(\alpha$ /Meff_G) [2, 44]. Finally, after adjusting, $P \leq$ $0.000001\left(-\log _{10}(P) \geq 6\right)$ was set as the critical threshold to detect SNPs significantly associated with the different root morphological traits.

\section{Candidate gene identification}

Candidate genes with SNP in coding regions and which could promote mutation were considered as high priority candidate genes. MaizeGDB (http://www. maizegdb.org/) and Gramene (https://www.gramene. org/) databases were used for predicting functional annotations of the candidate genes with reference to B73_RefGen_v4 [84, 85].

\section{Expression analysis}

High priority candidate genes were chosen based on the existence of substantial SNPs within the exon of candidate genes and phenotypic contribution rates greater than $12 \%$. For expression analysis, ten maize accessions with extreme root branching number differences from both GWAS and biparental populations were chosen. There were M08, M40, M25, M06, M35, M44, M63, M52, M56, and M54 from the GWAS population. From the mapping population, there were P014 (female parent), E1312 (male parent), C055, C039, C0143, C096, C004, C070, C152, and C010. The roots were sampled at two different stages (V1 and V3). Total RNA was extracted using the Trizol method, cDNA was reverse transcribed the All-in-One First-Strand cDNA Synthesis kit (GeneCopoeia Inc., USA) following the standard protocol. Oligonucleotide primers were designed with PrimerPremier5.0 (http://www.premierbiosoft.com) (Additional file 6: Table S5). The qRT-PCR protocol was performed in a total volume of $20 \mu \mathrm{L}: 2 \mu \mathrm{L}$ cDNA, $2 \mu \mathrm{L}$ of each forward and reverse primer, $10 \mu \mathrm{L}$ qPCR Master Mix, and $4 \mu \mathrm{L} d \mathrm{ddH}_{2} \mathrm{O}$. The qRT-PCR Thermo cycling conditions were: initial denaturation at $95{ }^{\circ} \mathrm{C}$ for $30 \mathrm{~s}, 40$ cycles of $95^{\circ} \mathrm{C}$ for $5 \mathrm{~s}$, annealing at $58^{\circ} \mathrm{C}$ for $30 \mathrm{~s}$, extension at $72{ }^{\circ} \mathrm{C}$ for $15 \mathrm{~s}$, and an infinite hold at $10{ }^{\circ} \mathrm{C}$. Leunig was used as the internal control gene and the relative expression levels were calculated using the $2^{\wedge}(-$ $\Delta \Delta \mathrm{ct}$ ) method [86]. All experiments were conducted in triplicates at each specified growth stage.

\section{Abbreviations \\ GWAS: Genome wide association study; QTL: Quantitative trait loci; SNP: Single nucleotide polymorphism; RDW: Root dry weight; RDW/ SDW: Root per shoot dry weight; TRL: Total root length; SUA: Surface area; ARD: Average root diameter; ROV: Root volume; RBN: Root branching number; SD: Standard deviation; CV: Coefficient of variation; $\mathrm{H}^{2}$ : Broad-sense heritability; LD: Linkage disequilibrium; EMMAX: Efficient mixed-model associ- ation expedited; MLM: Mixed linear model; FarmCPU: Fixed and random model circulating probability unification; GBS: Genotyping by sequencing; NGS: Next-generation sequencing; QQ plots: Quantile-quantile plots; RILs: Recombinant inbred lines; ANOVA: Analysis of variance; PCA: Principal component analysis}

\section{Supplementary Information}

The online version contains supplementary material available at https://doi. org/10.1186/s12864-021-07874-x

Additional file 1: Table S1. Phenotypic data of the seven root-related traits from the mapping population at V1, V2 and V3 stages.

Additional file 2: Table S2. Phenotypic data of the seven root-related traits from the GWAS panel at V1, V2 and V3 stages.

Additional file 3: Table S3. Physical positions of the identified QTLS. Additional file 4: Table S4. List of all significant SNPs detected in this study and their associated genes.

Additional file 5: Figure S1. Q-Q (quantile-quantile) plots of all traits at V1, V2 and V3 stages.

Additional file 6: Table S5. List of oligonucleotide primers used for the qRT-PCR assay for the evaluated candidate genes.

\section{Acknowledgements}

Authors would like to express their gratitude to Novogene Biological Company (Beijing, China) for their kind support in high throughput sequencing services. Authors also extend thanks to Dr. M.I. Nasser for his valuable help to improve this manuscript. 


\section{Authors' contributions}

AAM, AM, YJ, HZ, MZ, MASK, and QZ conducted the experiments. AAM and AM performed formal analysis. PW, SG and JQ supervised the experiments. AAM drafted the original manuscript. PW, YB, AL, MN, and GA reviewed and edited the manuscript. All authors have read and approved the final version of the manuscript.

\section{Funding}

This research was funded by the Modern Crop Seed Industry development of Jilin Province, China, (to Piwu Wang).

\section{Availability of data and materials}

All data analyzed during this study are included in the supplementary information files, and genotypic data have been deposited in the Sequence Read Archive (https://www.ncbi.nlm.nih.gov/sra) under the accession number PRJNA495031.

\section{Declarations}

\section{Ethics approval and consent to participate}

Not applicable.

\section{Consent for publication}

Not applicable.

\section{Competing interests}

The authors declare that they have no competing interests.

\section{Author details}

${ }^{1}$ College of Agronomy, Plant Biotechnology Center, Jilin Agricultural University, 130118 Changchun, Jilin, China. ${ }^{2}$ College of Life Sciences, Jilin Agricultural University, Jilin 130118 Changchun, China. ${ }^{3}$ Biology Department, Faculty of Sciences and Techniques, Abdou Moumouni University of Niamey, 10662 Niamey, Niger.

\section{Received: 9 November 2020 Accepted: 5 July 2021}

Published online: 20 July 2021

\section{References}

1. Ma J, Luo W, Zhang H, Zhou XH, Qin NN, Wei YM, Liu YX, Jiang QT, Chen $\mathrm{GY}$, Zheng $Y L$, et al. Identification of quantitative trait loci for seedling root traits from Tibetan semi-wild wheat (Triticum aestivum subsp. tibetanum). Genome. 2017:60(12):1068-75.

2. Pace J, Gardner C, Romay C, Ganapathysubramanian B, Lübberstedt T. Genome-wide association analysis of seedling root development in maize (Zea mays L.). BMC Genom. 2015;16(1):1-12.

3. Rogers ED, Benfey PN. Regulation of plant root system architecture: Implications for crop advancement. Curr Opin Biotechnol. 2015;32(Fig. 1): 93-8.

4. Ristova D, Busch W. Natural variation of root traits: from development to nutrient uptake. Plant Physiol. 2014;166(2):518-27.

5. Wasaya A, Zhang X, Fang Q, Yan Z. Root phenotyping for drought tolerance: a review. Agronomy. 2018;8(11):1-19.

6. Jackson MB, Armstrong W. Formation of aerenchyma and the processes of plant ventilation in relation to soil flooding and submergence. Plant Biol. 1999:1(3):274-287.

7. Uga Y, Sugimoto K, Ogawa S, Rane J, Ishitani M, Hara N, Kitomi Y, Inukai Y, Ono K, Kanno N, et al. Control of root system architecture by DEEPER ROOTING 1 increases rice yield under drought conditions. Nat Genet. 2013; 45(9):1097-1102.

8. Zaidi P, Seetharam K, Krishna G, Krishnamurthy L, Gajanan S, Babu R, Zerka M, Vinayan M, Vivek B. Genomic regions associated with root traits under drought stress in tropical maize (Zea mays L.). PloS one. 2016;11(10): e0164340.

9. Bruce W, Desbons P, Crasta O, Folkerts O. Gene expression profiling of two related maize inbred lines with contrasting root-lodging traits. J Exp Bot 2001;52(suppl_1):459-68

10. Price A, Tuberosa R, Dorlodot SD, Forster B. Root system architecture: opportunities and constraints for genetic improvement of crops. Trends Plant Sci. 2007;12(10):474-81.
11. Hirel B, Gouis JL, Ney B, Cyr RDS, Cedex V, Grignon T, Moulon F. The challenge of improving nitrogen use efficiency in crop plants: towards a more central role for genetic variability and quantitative genetics within integrated approaches. J Exp Bot. 2007:58(9):2369-87.

12. Garnett T, Conn V, Kaiser BN. Root based approaches to improving nitrogen use. Plant Cell Environ. 2009:32:1272-83.

13. Lynch JP. Steep, cheap and deep : an ideotype to optimize water and N acquisition by maize root systems. Ann Bot. 2013;112(2):347-57.

14. Marschener $\mathrm{H}$. Role of root growth, arbuscular mycorrhiza, and root exudates for the efficiency in nutrient acquisition. Field Crops Res. 1998;56: $203-7$.

15. Liu Z, Gao K, Shan S, Gu R, Wang Z, Craft EJ. Comparative Analysis of Root Traits and the Associated QTLs for Maize Seedlings Grown in Paper Roll, Hydroponics and Vermiculite Culture System. Front Plant Sci. 2017:8:1-13.

16. Peng Y, Niu J, Peng Z, Zhang F, Li C. Shoot growth potential drives N uptake in maize plants and correlates with root growth in the soil. Field Crops Res. 2010;115:85-93.

17. Abdel-Ghani AH, Kumar B, Reyes-Matamoros J, Gonzalez-Portilla PJ, Jansen C, Juan Pablo SM, Lee M, Lübberstedt T. Genotypic variation and relationships between seedling and adult plant traits in maize (Zea mays $L$.) inbred lines grown under contrasting nitrogen levels. Euphytica. 2013; 189(1):123-33.

18. Kumar B, Abdel-ghani AH, Pace J, Reyes-matamors J, Hochholdinger F, Lübberstedt T. Association analysis of single nucleotide polymorphisms in candidate genes with root traits in maize (Zea mays L.) seedlings. Plant Sci. 2014;224(July 2014):9-19.

19. Cai H, Chen F, Mi G, Zhang F, Maurer HP, Liu W, Reif JC, Yuan L. Mapping QTLs for root system architecture of maize (Zea mays $L$.) in the field at different developmental stages. Theor Appl Genet. 2012;125(6):1313-24.

20. Trachsel S, Kaeppler SM, Brown KM, Lynch JP. Shovelomics: High throughput phenotyping of maize (Zea mays L.) root architecture in the field. Plant Soil. 2011;341(1-2):75-87.

21. Gu D, Mei X, Yu T, Sun N, Xu D, Liu C, Cai Y. OTL identification for braceroot traits of maize in different generations and environments. Crop Sci. 2017;57(1):13-21.

22. Bray AL, Topp CN. The Quantitative Genetic Control of Root Architecture in Maize. Plant Cell Physiol. 2018:59(10):1919-30.

23. Fenta B, Beebe S, Kunert K, Burridge J, Barlow K, Lynch J, Foyer C. Field phenotyping of soybean roots for drought stress tolerance. Agronomy. 2014;4(3):418-35.

24. Ju C, Zhang W, Liu Y, Gao Y, Wang X, Yan J, Yang X. Genetic analysis of seedling root traits reveals the association of root trait with other agronomic traits in maize. BMC Plant Biol. 2018;18(171):1-15.

25. Zhu J, Kaeppler SM, Lynch JP. Mapping of QTLs for lateral root branching and length in maize (Zea mays L.) under differential phosphorus supply. Theor Appl Genet. 2005:111(4):688-95.

26. Laperche A, Devienne-Barret F, Maury O, Le Gouis J, Ney B. A simplified conceptual model of carbon/nitrogen functioning for QTL analysis of winter wheat adaptation to nitrogen deficiency. Theor Appl Genet. 2006;113(6): $1131-46$

27. Liu X, Li R, Chang X, Jing R. Mapping QTLs for seedling root traits in a doubled haploid wheat population under different water regimes. Euphytica. 2013;189(1):51-66

28. Ren Y, He X, Liu D, Li J, Zhao X, Li B, Tong Y, Zhang A, Li Z. Major quantitative trait loci for seminal root morphology of wheat seedlings. Mol Breeding. 2012;30(1):139-48.

29. Das A, Schneider H, Burridge J, Ascanio AKM, Wojciechowski T, Topp CN, Lynch JP, Weitz JS, Bucksch A. Digital imaging of root traits (DIRT): a highthroughput computing and collaboration platform for field-based root phenomics. Plant Methods. 2015;11(1):51.

30. Galkovskyi T, Mileyko Y, Bucksch A, Moore B, Symonova O, Price CA, Topp CN, lyer-Pascuzzi AS, Zurek PR, Fang S. GiA Roots: software for the high throughput analysis of plant root system architecture. BMC Plant Biol. 2012; 12(1):116

31. Pace J, Lee N, Naik HS, Ganapathysubramanian B, Lübberstedt T. Analysis of Maize (Zea mays L.) Seedling Roots with the High-Throughput Image Analysis Tool ARIA ( Automatic Root Image Analysis). PLoS ONE. 2014;9(9):1-10.

32. Pierret A, Gonkhamdee S, Jourdan C, Maeght J-L. IJ_Rhizo: an open-source software to measure scanned images of root samples. Plant Soil. 2013: 373(1-2):531-9. 
33. Rellán-Álvarez R, Lobet G, Lindner H, Pradier P-L, Sebastian J, Yee M-C, Geng Y, Trontin C, LaRue T, Schrager-Lavelle A. GLO-Roots: an imaging platform enabling multidimensional characterization of soil-grown root systems. Elife. 2015:4:e07597.

34. Symonova O, Topp CN, Edelsbrunner H. DynamicRoots: a software platform for the reconstruction and analysis of growing plant roots. PLoS One. 2015; 10(6):e0127657.

35. Lobet $G$, Pagès $L$, Draye $X$. A novel image-analysis toolbox enabling quantitative analysis of root system architecture. Plant Physiol. 2011;157(1): 29-39.

36. Arsenault J-L, Poulcur S, Messier C, Guay R. WinRHIZO'm , a root-measuring system with a unique overlap correction method. HortScience. 1995;30(4): 906D-906.

37. Abramoff M, Magelhaes $P$, Ram S. Image processing with Image. $J$ Biophotonics Intl. 2004; 11(7):36-42

38. Pound MP, French AP, Atkinson JA, Wells DM, Bennett MJ, Pridmore T. RootNav: navigating images of complex root architectures. Plant Physiol. 2013;162(4):1802-14

39. Leitner D, Felderer B, Vontobel P, Schnepf A. Recovering root system traits using image analysis exemplified by two-dimensional neutron radiography images of lupine. Plant Physiol. 2014;164(1):24-35.

40. Clark RT, Famoso AN, Zhao K, Shaff JE, Craft EJ, Bustamante CD, McCouch SR, Aneshansley DJ, Kochian LV. High-throughput two-dimensional root system phenotyping platform facilitates genetic analysis of root growth and development. Plant Cell Environ. 2013;36(2):454-66.

41. Watt M, Moosavi S, Cunningham SC, Kirkegaard J, Rebetzke G, Richards R. A rapid, controlled-environment seedling root screen for wheat correlates well with rooting depths at vegetative, but not reproductive, stages at two field sites. Ann Botany. 2013;112(2):447-55.

42. Li C, Fu Y, Sun R, Wang Y, Wang Q. Single-locus and multi-locus genomewide association studies in the genetic dissection of fiber quality traits in upland cotton (Gossypium hirsutum L.). Front Plant Sci. 2018;9:1083.

43. Khan SU, Yangmiao J, Liu S, Zhang K, Khan MHU, Zhai Y, Olalekan A, Fan C, Zhou Y. Genome-wide association studies in the genetic dissection of ovule number, seed number, and seed weight in Brassica napus L. Industr Crops Prod. 2019;142:111877.

44. Sanchez DL, Liu S, Ibrahim R, Blanco M, Lübberstedt T. Genome-wide association studies of doubled haploid exotic introgression lines for root system architecture traits in maize (Zea mays L.). Plant Sci. 2018;268:30-8.

45. Moussa AA, Mandozai A, Qu J, Jin Y, Zhang Q, Abd El-Rahim MG, Anwari G, Sharaf A, Wang P. Mapping QTLs using high-density snps genotyped by sequencing reveals novel potential regions underlying maize root morphological traits at seedling stage. Int J Agri Biol. 2021;25(4):904-14.

46. Abdel AH, Bharath $G$, Jordon $K$, Constantin P, Gonzalez PJ, Jenaro P, Matamoros R, Pablo J, Martin S, Lee M, et al. Association analysis of genes involved in maize (Zea mays L.) root development with seedling and agronomic traits under contrasting nitrogen levels. Plant Mol Biol. 2015; 88(1-2):133-47.

47. Pace J, Yu X, Thomas L. Genomic prediction of seedling root length in maize (Zea mays L.). Plant J. 2015;83(5):903-12.

48. Comas L, Becker S, Cruz VMV, Byrne PF, Dierig DA. Root traits contributing to plant productivity under drought. Front Plant Sci. 2013;4:442.

49. Song W, Wang B, Hauck AL, Dong X, Li J, Lai J. Genetic dissection of maize seedling root system architecture traits using an ultra-high density bin-map and a recombinant inbred line population. J Integr Plant Biol. 2016;58(3): 266-79.

50. Crowell S, Korniliev P, Falcao A, Ismail A, Gregorio G, Mezey J, McCouch S. Genome-wide association and high-resolution phenotyping link Oryza sativa panicle traits to numerous trait-specific QTL clusters. Nat Commun. 2016;7(1):1-14

51. Lu S, Zhang M, Zhang Z, Wang Z, Wu N, Song Y, Wang P. Screening and verification of genes associated with leaf angle and leaf orientation value in inbred maize lines. PLOS ONE. 2018;13(12):1-16.

52. Dai L, Wu L, Dong Q, Zhang Z, Wu N, Song Y, Lu S, Wang P. Genome-wide association study of field grain drying rate after physiological maturity based on a resequencing approach in elite maize germplasm. Euphytica. 2017;213(8):182.

53. Romay MC, Millard MJ, Glaubitz JC, Peiffer JA, Swarts KL, Casstevens TM, Elshire RJ, Acharya CB, Mitchell SE, Flint-Garcia SA. Comprehensive genotyping of the USA national maize inbred seed bank. Genome Biol. 2013;14(6):R55.
54. Dhanapal AP, Crisosto $\mathrm{CH}$. Association genetics of chilling injury susceptibility in peach (Prunus persica (L.) Batsch) across multiple years. 3 Biotech. 2013;3(6):481-90.

55. Hecht BC, Campbell NR, Holecek DE, Narum SR. Genome-wide association reveals genetic basis for the propensity to migrate in wild populations of rainbow and steelhead trout. Mol Ecol. 2013;22(11):3061-76.

56. Zhang H, Fan X, Zhang Y, Jiang J, Liu C. Identification of favorable SNP alleles and candidate genes for seedlessness in Vitis vinifera $L$. using genome-wide association mapping. Euphytica. 2017;213(7):136.

57. Hund A, Reimer R, Messmer R. A consensus map of QTLs controlling the root length of maize. Plant Soil. 2011;344(1):143-58.

58. Winter D, Vinegar B, Nahal H, Ammar R, Wilson GV, Provart NJ. An "Electronic Fluorescent Pictograph" browser for exploring and analyzing large-scale biological data sets. PloS one. 2007;2(8):e718.

59. Hoopes GM, Hamilton JP, Wood JC, Esteban E, Pasha A, Vaillancourt B, Provart NJ, Buell CR. An updated gene atlas for maize reveals organ-specific and stress-induced genes. Plant J. 2019;97(6):1154-67.

60. Stelpflug SC, Sekhon RS, Vaillancourt B, Hirsch CN, Buell CR, de Leon N, Kaeppler SM. An expanded maize gene expression atlas based on RNA sequencing and its use to explore root development. Plant Genome. 2016; 9(1):1-16.

61. Sekhon RS, Lin H, Childs KL, Hansey CN, Buell CR, De Leon N, Kaeppler SM. Genome-wide atlas of transcription during maize development. Plant J. 2011;66(4):553-63.

62. Li L, Hey S, Liu S, Liu Q, McNinch C, Hu H-C, Wen T-J, Marcon C, Paschold A, Bruce W. Characterization of maize roothairless 6 which encodes a D-type cellulose synthase and controls the switch from bulge formation to tip growth. Sci Rep. 2016;6(1):1-12.

63. Nestler J, Liu S, Wen TJ, Paschold A, Marcon C, Tang HM, Li D, Li L, Meeley RB, Sakai H. Roothairless5, which functions in maize ( $Z$ ea mays L.) root hair initiation and elongation encodes a monocot-specific NADPH oxidase. Plant J. 2014;79(5):729-40.

64. von Behrens I, Komatsu M, Zhang Y, Berendzen KW, Niu X, Sakai H, Taramino G, Hochholdinger F. Rootless with undetectable meristem 1 encodes a monocot-specific AUX/IAA protein that controls embryonic seminal and post-embryonic lateral root initiation in maize. Plant J. 2011; 66(2):341-53.

65. Woll K, Borsuk LA, Stransky H, Nettleton D, Schnable PS, Hochholdinger F. Isolation, characterization, and pericycle-specific transcriptome analyses of the novel maize lateral and seminal root initiation mutant rum1. Plant Physiol. 2005;139(3):1255-67.

66. Wen TJ, Schnable PS. Analyses of mutants of three genes that influence root hair development in Zea mays (Gramineae) suggest that root hairs are dispensable. Am J Bot. 1994;81(7):833-42.

67. Zhang S, Chen X, Lu C, Ye J, Zou M, Lu K, Feng S, Pei J, Liu C, Zhou X. Genome-wide association studies of 11 agronomic traits in cassava (Manihot esculenta Crantz). Front Plant Sci. 2018:9:503.

68. Bustin S. Invited review quantification of mRNA using real-time reverse transcription PCR (RT-PCR): trends and problems. J Mol Endocrinol. 2002;29:23-39.

69. Bustin SA, Nolan T. Pitfalls of quantitative real-time reverse-transcription polymerase chain reaction. J Biomol Tech. 2004;15(3):155.

70. Mandozai A, Abdourazak AM, Qi Z, Qu J, Ahmad N, Du Y, al Amin N, Theorent R, Anwari G, Wang P. Evaluation and clustering of soybean (Glycine max L.) germplasm inbred lines for root and shoot morphological traits at seedling stage. Int J Biosci. 2019;15(6):152-63.

71. Wu Y, Bhat PR, Close TJ, Lonardi S. Efficient and accurate construction of genetic linkage maps from the minimum spanning tree of a graph. PLoS Genet. 2008;4(10):e1000212.

72. Meng L, Li H, Zhang L, Wang JJTCJ. QTL IciMapping: integrated software for genetic linkage map construction and quantitative trait locus mapping in biparental populations. Crop J. 2015;3(3):269-83.

73. Wang J, Li H, Zhang L, Meng L: User's manual of QTL IciMapping ver. 4.1. The Quantitative Genetics Group, Institute of Crop Science, Chinese Academy of Agricultural Sciences (CAAS), Beijing and Genetic Resources Program, International Maize and Wheat Improvement Center (CIMMYT), Apdo. Mexico. 2016.

74. Li F, Jia H, Liu L, Zhang C, Liu Z, Zhang Z. Quantitative trait loci mapping for kernel row number using chromosome segment substitution lines in maize. Genet Mol Res. 2014;13(1):1707-16.

75. Murray M, Thompson W. Protocol of DNA isolation. Nucl Acids Res. 1980;8: 4321-5. 
76. Li H, Durbin R. Fast and accurate short read alignment with BurrowsWheeler transform. Bioinformatics. 2009;25(14):1754-60.

77. Li H, Wysoker A, Durbin R. 1000 Genome project data processing subgroup. The sequence alignment/Map format and SAMtools. Bioinformatics. 2009; 25(16):2078-9.

78. Yang J, Lee SH, Goddard ME, Visscher PM. GCTA: a tool for genome-wide complex trait analysis. Am J Hum Genet. 2011;88(1):76-82.

79. Purcell S, Neale B, Todd-Brown K, Thomas L, Ferreira MA, Bender D, Maller J, Sklar P, De Bakker PI, Daly MJ. PLINK: a tool set for whole-genome association and population-based linkage analyses. Am J Hum Genet. 2007; 81(3):559-75.

80. Yu J, Pressoir G, Briggs WH, Bi IV, Yamasaki M, Doebley JF, McMullen MD, Gaut BS, Nielsen DM, Holland JB. A unified mixed-model method for association mapping that accounts for multiple levels of relatedness. Nat Genet. 2006;38(2):203-8.

81. Kang HM, Sul JH, Service SK, Zaitlen NA, Kong S-y, Freimer NB, Sabatti C, Eskin E. Variance component model to account for sample structure in genome-wide association studies. Nat Genet. 2010;42(4):348-54.

82. Liu X, Huang M, Fan B, Buckler ES, Zhang Z. Iterative usage of fixed and random effect models for powerful and efficient genome-wide association studies. PLoS Genet. 2016;12(2):e1005767.

83. Bradbury PJ, Zhang Z, Kroon DE, Casstevens TM, Ramdoss Y, Buckler ES. TASSEL: software for association mapping of complex traits in diverse samples. Bioinformatics. 2007;23(19):2633-5.

84. Liu N, Xue Y, Guo Z, Li W, Tang J. Genome-wide association study identifies candidate genes for starch content regulation in maize kernels. Front Plant Sci. 2016;7:1046.

85. Schnable PS, Ware D, Fulton RS, Stein JC, Wei F, Pasternak S, Liang C, Zhang J, Fulton L, Graves TA. The B73 maize genome: complexity, diversity, and dynamics. Science. 2009;326(5956):1112-5.

86. Livak KJ, Schmittgen TD. Analysis of relative gene expression data using real-time quantitative PCR and the $2-\triangle \Delta C T$ method. Methods. 2001;25(4): 402-8.

\section{Publisher's Note}

Springer Nature remains neutral with regard to jurisdictional claims in published maps and institutional affiliations.

Ready to submit your research? Choose BMC and benefit from:

- fast, convenient online submission

- thorough peer review by experienced researchers in your field

- rapid publication on acceptance

- support for research data, including large and complex data types

- gold Open Access which fosters wider collaboration and increased citations

- maximum visibility for your research: over $100 \mathrm{M}$ website views per year

At $\mathrm{BMC}$, research is always in progress.

Learn more biomedcentral.com/submissions 